\title{
A multiwavelength study of the massive star-forming region IRAS 06055+2039 (RAFGL 5179)
}

\author{
A. Tej ${ }^{1}$, D. K. Ojha ${ }^{1}$, S. K. Ghosh ${ }^{1}$, V. K. Kulkarni ${ }^{2}$, R. P. Verma ${ }^{1}$, S. Vig ${ }^{1}$, and T. P. Prabhu ${ }^{3}$ \\ 1 Tata Institute of Fundamental Research, Mumbai 400 005, India \\ e-mail: tej@tifr.res.in \\ 2 National Centre for Radio Astrophysics, Post Bag 3, Ganeshkhind, Pune 411 007, India \\ 3 Indian Institute of Astrophysics, Koramangala, Bangalore 560 034, India
}

Received 14 December 2005 / Accepted 17 January 2006

ABSTRACT

\begin{abstract}
Aims. We present a multiwavelength study of the massive star-forming region associated with IRAS 06055+2039.
Methods. Narrow-band near-infrared (NIR) observations were carried out with UKIRT-UFTI in molecular hydrogen and Br $\gamma$ lines to trace the shocked and ionized gases, respectively. We have used 2MASS $J H K_{\mathrm{s}}$ data to study the nature of the embedded cluster associated with IRAS $06055+2039$. The radio emission from the ionized gas was mapped at 610 and $1280 \mathrm{MHz}$ using the Giant Metrewave Radio Telescope (GMRT), India. Emission from warm dust and the unidentified infrared bands (UIBs) was estimated using the mid-infrared $(8-21 \mu \mathrm{m})$ data from the MSX survey. Submillimetre emission from the cold dust at 450 and $850 \mu \mathrm{m}$ was studied using JCMT-SCUBA.

Results. For the infrared cluster associated with IRAS $06055+2039$, we obtain a power-law slope of $0.43 \pm 0.09$ for the $K_{\mathrm{s}}$-band luminosity function (KLF), which is in good agreement with other young embedded clusters. We estimate an age of 2-3 Myr for this cluster. Apart from the diffuse emission, the high-resolution $1280 \mathrm{MHz}$ map also shows the presence of several discrete sources that possibly represent high-density clumps. The morphology of shocked molecular hydrogen forms an arc towards the N-E of the central IRAS point source and envelopes the radio emission. Submillimetre emission shows the presence of a dense cloud core that is probably at an earlier evolutionary stage compared to the ionized region with shocked molecular gas lying between the two. The total mass of the cloud is estimated to be $\sim 7000-9000 M_{\odot}$ from the submillimetre emission at 450 and $850 \mu \mathrm{m}$.

Conclusions. The multiwavelength study of this star-forming complex reveals an interesting scenario where regions are at different stages in the evolution of star formation.
\end{abstract}

Key words. infrared: ISM - radio continuum: ISM - ISM: H II regions - ISM: individual objects: IRAS 06055+2039

\section{Introduction}

Massive stars are preferentially formed in dense cores of molecular clouds. They remain deeply embedded in the prenatal molecular gas and obscuring dust and their pre-main-sequence (PMS) time scales are much shorter compared to the low mass stars. The luminous high-mass stars also affect the parent cloud. In addition, massive stars do not form in isolation but often in clusters and associations. All these factors contribute towards making the study of the formation mechanisms of these systems very difficult. Multiwavelength studies, therefore, hold the potential to probe these complexes at different depths and unravel the least understood aspects of massive star formation.

IRAS 06055+2039(G189.78+0.34, RAFGL 5179) is a massive star-forming region chosen from the catalog of massive young stellar objects by Chan et al. (1996). G189.78+0.34 is listed as an ultracompact (UC) HII region (Shepherd \& Churchwell 1996; Bronfman et al. 1996). It belongs to the Gem OB1 molecular cloud complex and is a part of the extended HII region Sh 252. It is associated with S252 A, which is one of the six compact radio sources in Sh 252 revealed from the $5 \mathrm{GHz}$ aperture synthesis observations by Felli et al. (1977). IRAS fluxes yield a far-infrared luminosity of $\sim 10^{4} L_{\odot}$ (Carpenter et al. 1995) for this star-forming region. There are several kinematic distance estimates used in the literature for this source, and they range from $1.25 \mathrm{kpc}$ (Mirabel et al. 1987) to $2.9 \mathrm{kpc}$ (Wouterloot \& Brand 1989). In this paper we use the value of $2.6 \mathrm{kpc}$ (Wu et al. 2001), which is the most widely used distance estimate for this source.

This high-mass star-forming region has been observed as part of many surveys. The $\mathrm{H}_{2} \mathrm{O}$ maser (Kömpe et al. 1989; Lada \& Wooden 1979) and the $6.7 \mathrm{GHz}$ methanol maser (Szymczak et al. 2000a) have been detected towards IRAS 06055+2039. Positive detection has also been made in $\mathrm{SiO}$ (Harju et al. 1998), CO (2-1) (Wu et al. 2001), and CS (2-1) (Bronfman et al. 1996). Zinchenko et al. (1998) in their study of dense molecular cores have also mapped this source in CS (2-1). The CO maps of Shepherd \& Churchwell (1996) do not show evidence of any bipolar outflows. Searches for the $6 \mathrm{~cm}$ (Szymczak et al. 2000b) and $5 \mathrm{~cm}$ (Baudry et al. 1997) $\mathrm{OH}$ masers show negative results. The IRAS low-resolution spectra show a relatively red continuum from 13 to $23 \mu \mathrm{m}$ with the presence of an emission feature at $11.3 \mu \mathrm{m}$ that is attributed to the presence of polycyclic aromatic hydrocarbon (PAH) molecules (Kwok et al. 1997).

In this paper, we present a multiwavelength study of this star-forming region. In Sect. 2, we present the narrow-band nearinfrared (NIR) and radio continuum observations and a brief description of the related data reduction procedures. In Sect. 3, we discuss other available datasets used in the present study. 

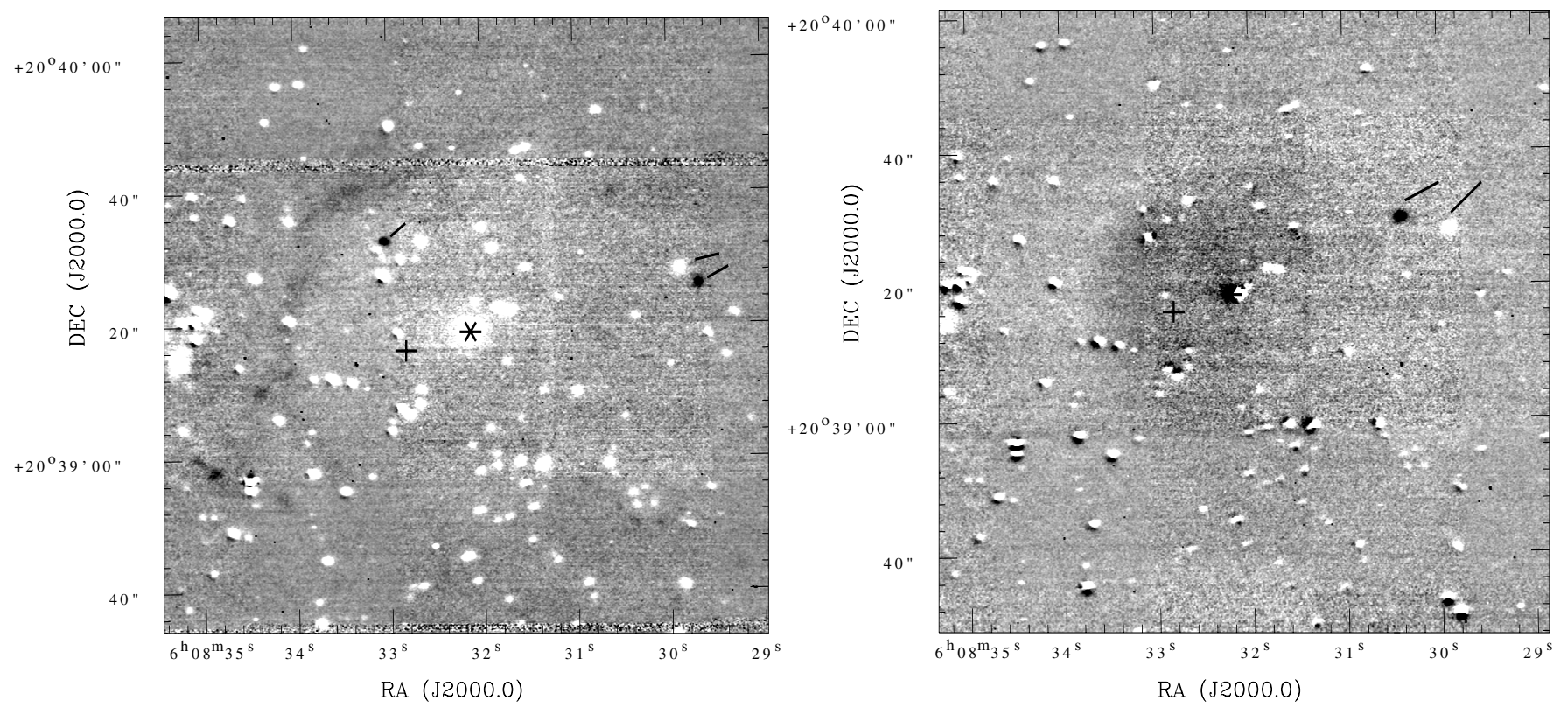

Fig. 1. The continuum-subtracted $\mathrm{H}_{2}(1-0) \mathrm{S} 1$ (left) and $\mathrm{Br} \gamma$ (right) images of the field around IRAS 06055+2039. The images were smoothed to a resolution of $0.3^{\prime \prime} \times 0.3^{\prime \prime}(3 \times 3$ box $)$ to improve the contrast of the faint features. Residuals of continuum subtraction around stars are seen in both images. The plus sign and the asterisk in both images mark the position of the IRAS point source and the central, brightest star in the NIR cluster, respectively. Artifacts (possibly ghost images of the central, bright star) are seen in $\mathrm{H}_{2}, \mathrm{Br} \gamma$, and the $K$ continuum frames. The resulting residuals in both the continuum-subtracted images are indicated with lines.

Section 4 gives a comprehensive discussion of the results obtained and in Sect. 5, we summarise the results.

\section{Observations and data reduction}

\subsection{Near-infrared observations}

Narrow-band NIR observations were carried out in the rotational-vibrational line of molecular hydrogen $-\mathrm{H}_{2}(1-0) \mathrm{S} 1$ $(\lambda=2.12 \mu \mathrm{m}, \Delta \lambda=0.02 \mu \mathrm{m})$, hydrogen recombination line of $\operatorname{Br} \gamma(\lambda=2.16 \mu \mathrm{m}, \Delta \lambda=0.02 \mu \mathrm{m})$, and $K$ continuum $(\lambda=2.27 \mu \mathrm{m}, \Delta \lambda=0.02 \mu \mathrm{m})$ at the $3.8 \mathrm{~m}$ United Kingdom Infrared Telescope (UKIRT ${ }^{1}$ ), Hawaii. Observations were carried out on 16 Feb. 2002 under the UKIRT Service Programme (Proposal No. 1459). The instrument used was the UKIRT Fast Track Imager (UFTI), which is a $1-2.5 \mu \mathrm{m}$ camera with a $1024 \times 1024 \mathrm{HgCdTe}$ array having a plate scale of $0.091^{\prime \prime}$ per pixel. The "JITTER-SELF-FLAT" data reduction (DR) recipe was used. This script takes imaging observations comprising 9 jittered object frames and a dark frame. A flat field is then created from the sequence of jittered object frames. The final image is a mosaic generated from the 9 frames after dark subtraction and flat fielding, and it has a total field of view of $\sim 4 \times 4 \mathrm{arcmin}^{2}$. For our observations, the integration time was $100 \mathrm{~s}$ in each band. To obtain pure emission line images, it is essential to subtract out the contribution from the continuum. This is done by subtracting the $K$ continuum image from the $\mathrm{H}_{2}$ and the $\mathrm{Br} \gamma$ images after proper alignment and PSF matching. Figure 1 shows the continuum-subtracted $\mathrm{H}_{2}$ and $\mathrm{Br} \gamma$ images of the central field around IRAS $06055+2039$. The continuum-subtracted $\mathrm{H}_{2}$ image displays a prominent arc towards the N-E of the central source. The continuum-subtracted $\operatorname{Br} \gamma$ image shows the

1 The United Kingdom Infrared Telescope is operated by the Joint Astronomy Centre on behalf of the UK Particle Physics and Astronomy Research Council. presence of faint, diffuse emission surrounding the central, bright source.

\subsection{Radio continuum observations}

In order to probe the ionized gas component, radio continuum interferometric mapping of the region around IRAS $06055+2039$ was carried out using the Giant Metrewave Radio Telescope (GMRT) array, India. The GMRT has a "Y"-shaped hybrid configuration of 30 antennas, each $45 \mathrm{~m}$ in diameter. There are six antennas along each of the three arms (with arm length of $\sim 14 \mathrm{~km}$ ). These provide high angular resolution (longest baseline $\sim 25 \mathrm{~km}$ ). The rest of the twelve antennas are located in a random and compact $1 \times 1 \mathrm{~km}^{2}$ arrangement near the centre and is sensitive to large scale diffuse emission (shortest baseline $\sim 100 \mathrm{~m}$ ). Details of the GMRT antennae and their configurations can be found in Swarup et al. (1991). Observations were carried out at 1280 and $610 \mathrm{MHz}$. The radio sources 3C 48 and 3C 147 were used as the primary flux calibrators, while $0532+194$ and $0432+416$ were used as phase calibrators for the 1280 and $610 \mathrm{MHz}$ observations, respectively.

Data was reduced using AIPS. The data sets were carefully checked using tasks UVPLT and VPLOT for bad data (owing to dead antennae, bad baselines, interference, spikes, etc.). Subsequent editing was carried out using the tasks UVFLG and TVFLG. Maps of the field were generated by Fourier inversion and subsequent cleaning using the task IMAGR. Several iterations of self calibration were carried out to obtain improved maps.

Figure 2 shows the radio continuum images at 1280 and

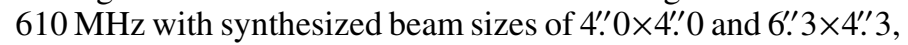
respectively. The rms noises in the maps are $0.3(1280 \mathrm{MHz})$ and $0.4(610 \mathrm{MHz}) \mathrm{mJy} / \mathrm{beam}$. Table 1 gives the details of the observations and the maps. 

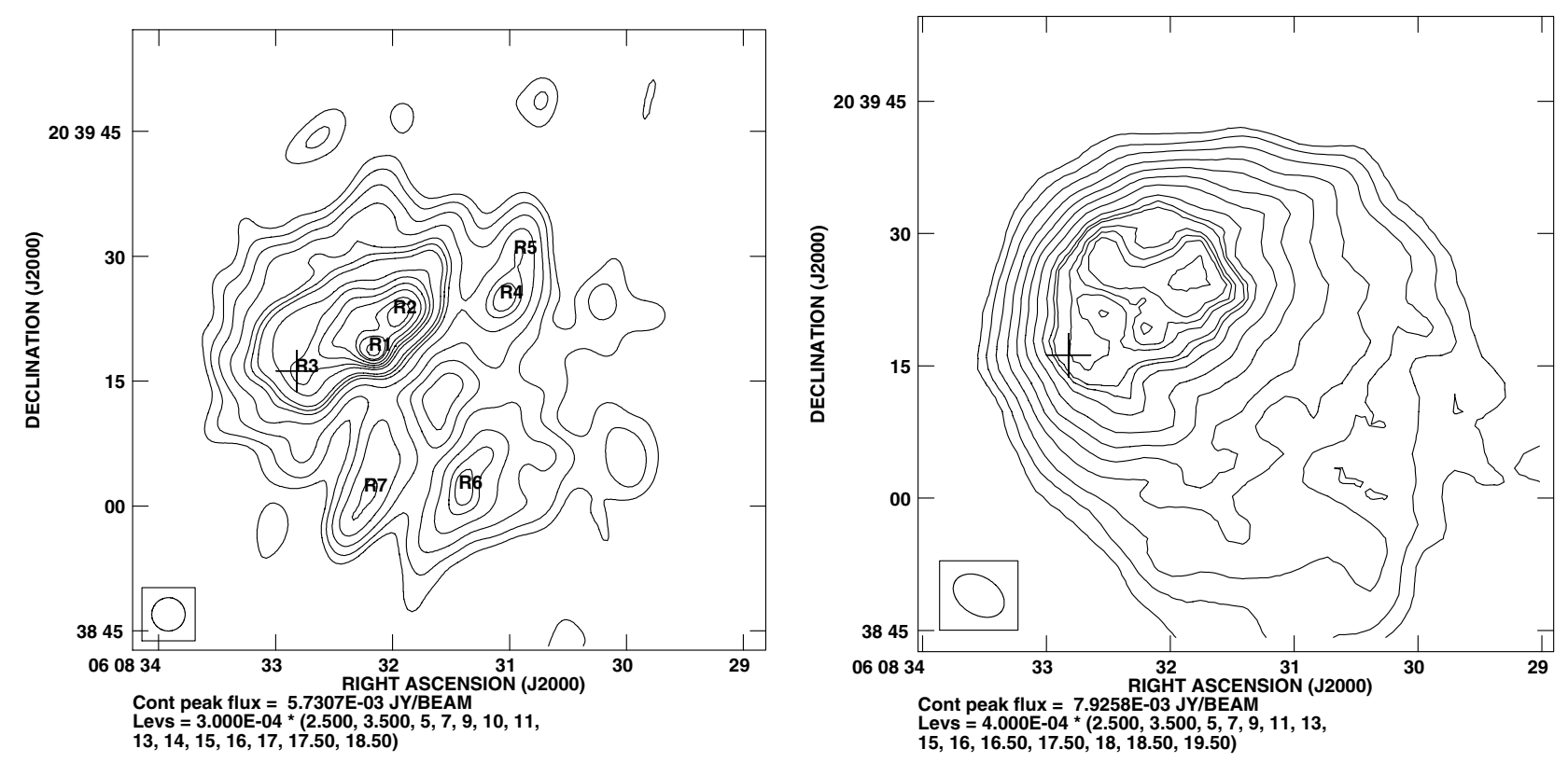

Fig. 2. High-resolution radio continuum maps at $1280 \mathrm{MHz}$ (left) and $610 \mathrm{MHz}$ (right) for the region around IRAS 06055+2039. The numbers R1, $\mathrm{R} 2, \ldots . . \mathrm{R} 7$ mark the position of discrete point sources (see text). The rms noises at 1280 and $610 \mathrm{MHz}$ are $\sim 0.3$ and $0.4 \mathrm{mJy} / \mathrm{beam}$, respectively. The plus sign in each image marks the IRAS point source position.

Table 1. Details of the radio interferometric continuum observations.

\begin{tabular}{lcc}
\hline \hline Details & $1280 \mathrm{MHz}$ & $610 \mathrm{MHz}$ \\
\hline Date of Obs. & 3 Aug. 2003 & 21 May 2002 \\
Primary beam & $26^{\prime}$ & $54^{\prime}$ \\
Cont. bandwidth (MHz) & 16 & 16 \\
Synth. beam & $4^{\prime \prime} 0 \times 44^{\prime \prime} 0$ & $66^{\prime \prime} 3 \times 4^{\prime \prime} 3$ \\
Position angle. (deg) & - & 58 \\
Peak Flux (mJy/beam) & 5.7 & 7.9 \\
rms noise (mJy/beam) & 0.3 & 0.4 \\
\hline
\end{tabular}

\section{Other available datasets}

\subsection{Near-infrared data from 2 MASS}

NIR $\left(J H K_{\mathrm{s}}\right)$ data for point sources around IRAS $06055+2039$ have been obtained from the Two Micron All Sky Survey ${ }^{2}$ (2MASS) Point Source Catalog (PSC). Source selection was based on the "read-flag" that gives the uncertainties in the magnitudes. In our sample we retain only those sources for which the "read-flag" values are $1-6$. The 2MASS data were used to study the embedded cluster associated with IRAS 06055+2039.

\subsection{Mid-infrared data from MSX}

The Midcourse Space Experiment ${ }^{3}$ (MSX) surveyed the Galactic plane in four mid-infrared bands - A (centre $\lambda: 8.28 \mu \mathrm{m}$;

2 This publication makes use of data products from the Two Micron All Sky Survey, which is a joint project of the University of Massachusetts and the Infrared Processing and Analysis Center/California Institute of Technology, funded by the NASA and the NSF.

3 This research made use of data products from the Midcourse Space Experiment. Processing of the data was funded by the Ballistic Missile Defense Organization with additional support from NASA Office of Space Science. This research also made use of the NASA/ IPAC Infrared Science Archive, which is operated by the Jet Propulsion Laboratory, Caltech, under contract with the NASA.
$50 \%$ peak intensity range: $6.8-10.8 \mu \mathrm{m}), \mathrm{C}(12.13 \mu \mathrm{m}$; 11.1-13.2 $\mu \mathrm{m}), \mathrm{D}(14.65 \mu \mathrm{m} ; 13.5-15.9 \mu \mathrm{m})$, and $\mathrm{E}(21.34 \mu \mathrm{m}$; $18.2-25.1 \mu \mathrm{m}$ ) at a spatial resolution of $\sim 18^{\prime \prime}$ (Price et al. 2001). Two of these bands (A and C) cover the UIBs at 6.2, 7.7, 8.7, 11.3, and $12.7 \mu \mathrm{m}$. The integrated flux densities of IRAS $06055+2039$ in these bands are listed in Table 2. The MSX images in these four bands for the region around IRAS $06055+2039$ were used to study the emission from the UIBs and to estimate the spatial distribution of temperature and optical depth of the warm interstellar dust.

\subsection{Mid- and far-infrared data from IRAS}

The data from the IRAS survey in the four bands $(12,25,60$, and $100 \mu \mathrm{m}$ ) for the region around IRAS 06055+2039 were HIRESprocessed (Aumann et al. 1990) to obtain high angular resolution maps. These maps were used to determine the spatial distribution of dust colour temperature and optical depth. The integrated flux densities from the HIRES-processed images and IRAS-PSC are also given in Table 2.

\subsection{Sub-mm data from JCMT}

Submillimetre observations using the Submillimetre CommonUser Bolometer Array (SCUBA) instrument of the James Clerk Maxwell Telescope ${ }^{4}$ (JCMT) were retrieved from the JCMT archives and processed using their standard pipeline SCUBA User Reduction Facility (SURF). JCMT-SCUBA observations for the data used in our study were carried out on 25 Oct. 2000. Uranus was used as the primary flux calibrator for the maps. Figure 3 displays the spatial distribution of cold dust emission

4 This paper makes use of data from the James Clerk Maxwell Telescope Archive. The JCMT is operated by the Joint Astronomy Centre on behalf of the UK particle Physics and Astronomy Research Council, the National Research Council of Canada, and the Netherlands Organisation for Pure Research. 
Table 2. Infrared flux densities for IRAS 06055+2039.

\begin{tabular}{lccccccccc}
\hline \hline & \multicolumn{3}{c}{ MSX $^{a}$ images } & \multicolumn{5}{c}{ IRAS images } \\
\hline Wavelength $(\mu \mathrm{m})$ & 8.3 & 12.1 & 14.7 & 21.3 & & 12 & 25 & 60 & 100 \\
Flux density (Jy) & 26 & 34 & 24 & 68 & PSC & 15.6 & 76.8 & 1032 & 1715 \\
& & & & & HIRES $^{a}$ & 38 & 113 & 1280 & 1691 \\
\hline
\end{tabular}

${ }^{a}$ Flux densities were obtained by integrating over a circular region of diameter $3^{\prime}$ centred on the peak.
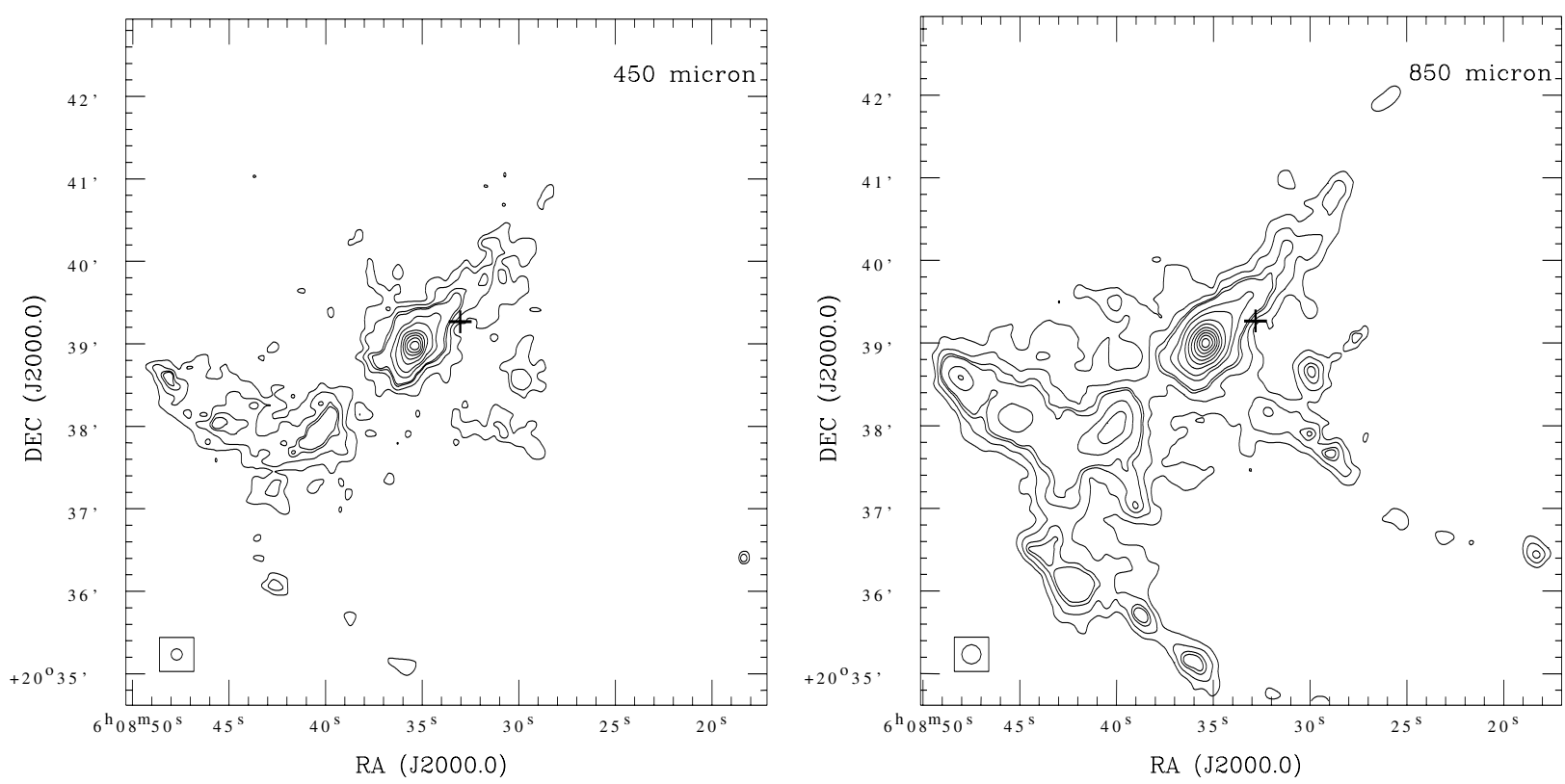

Fig. 3. Contour maps showing the spatial distribution of dust emission at $450 \mu \mathrm{m}$ (left) and $850 \mu \mathrm{m}($ right $)$ for the region around IRAS $06055+2039$. The contour levels are at 5, 7, 9, 10, 15, 20,30,40,50,60,70,80, and 90\% of the peak value of 29.45 and $6.83 \mathrm{Jy} / \mathrm{beam}$ at 450 and $850 \mu \mathrm{m}$, respectively. The FWHMs of the symmetric 2D Gaussian beams are $7{ }^{\prime \prime} .8$ and $15^{\prime \prime} .2$ for the two wave bands. The plus sign in each image marks the position of the IRAS point source.

at 450 and $850 \mu \mathrm{m}$. Large atmospheric extinction correction was applied to obtain the $450 \mu \mathrm{m}$ map.

\section{Results and discussion}

\subsection{Embedded cluster in the near-infrared}

\subsubsection{Radial profile and stellar surface number density}

The 2MASS $K_{\mathrm{s}}$-band image of the region around IRAS $06055+2039$ is shown in Fig. 4. We see the presence of a diffuse emission region harbouring an infrared cluster.

This cluster is also listed in the catalog of embedded infrared clusters compiled by Bica et al. (2003). We use the 2MASS $J H K_{\mathrm{s}}$ data to study the nature of this cluster. For determining the $K_{\mathrm{s}}$-band radial profile and the stellar surface number density (SSND), we selected sources that are detected in the $K_{\mathrm{S}}$ band. In order to estimate the cluster radius, we selected a large region of radius 300" centred on IRAS $06055+2039$ $\left(\alpha_{2000.0}=06^{\mathrm{h}} 08^{\mathrm{m}} 32^{\mathrm{s}} .1 ; \delta_{2000.0}=+20^{\circ} 39^{\prime} 18^{\prime \prime}\right)$. To account for the contribution from the field stars, we selected a control field $\left(\alpha_{2000.0}=06^{\mathrm{h}} 09^{\mathrm{m}} 52^{\mathrm{s}} .0 ; \delta_{2000.0}=+20^{\circ} 39^{\prime} 18^{\prime \prime}\right)$ which is $\sim 20^{\prime}$ to the east of IRAS $06055+2039$. Figure 5 shows the radial profile of the stellar density in log-log scale. This profile was created by counting the number of stars in $10^{\prime \prime}$ annuli and normalizing by the annulus area. We fitted two models to the surface density radial profile - the King's profile and the inverse radius $\left(r^{-1}\right)$

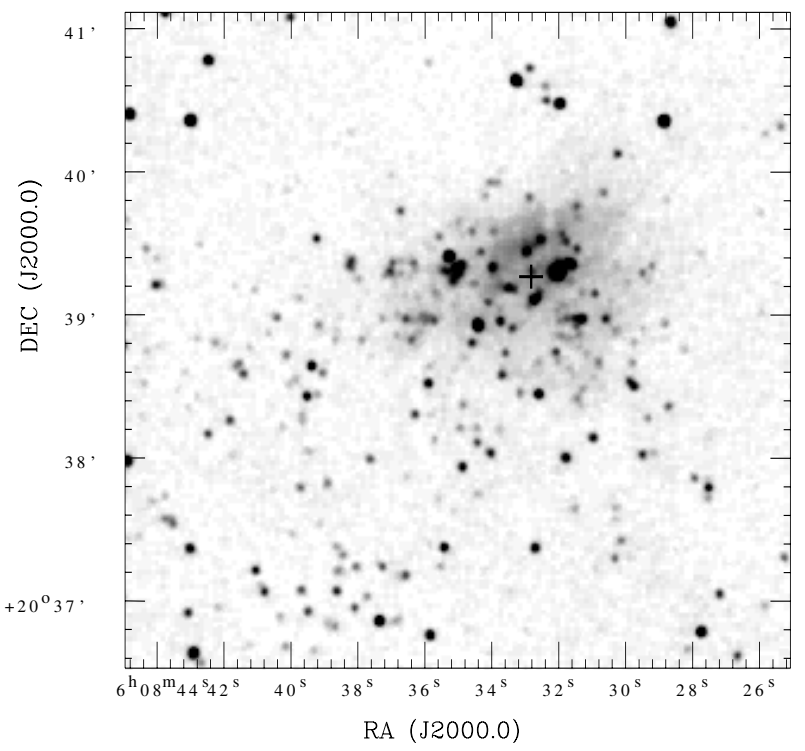

Fig. 4. The 2MASS $K_{\mathrm{s}}$-band image of the region around IRAS $06055+2039$. The presence of a diffuse emission region and an NIR cluster is seen. The plus sign marks the position of the IRAS point source.

model. Neglecting the tidal radius, the King's profile can be written as

$f(r)=a+\frac{f_{0}}{1+\left(r / r_{\mathrm{c}}\right)^{2}}$ 


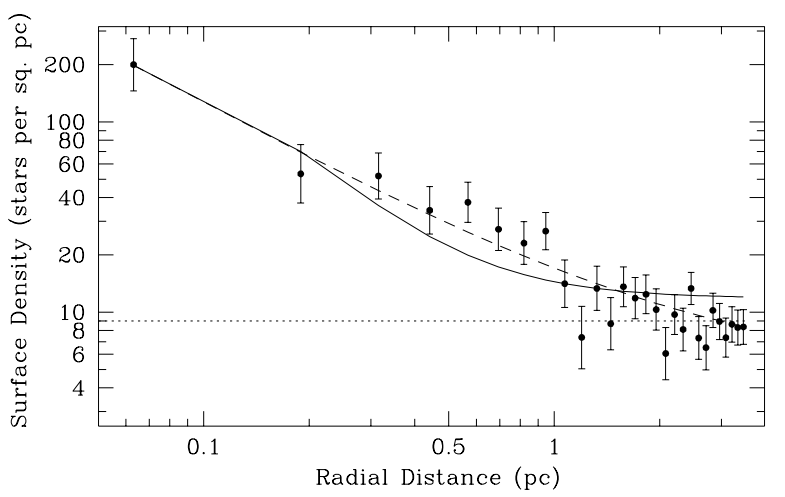

Fig. 5. The radial profile of the surface number density for the cluster associated with IRAS $06055+2039$ in log-log scale. Also plotted are the two fitted models - King's model (solid) and inverse radius model (dashed). As compared to the King's model, the inverse radius model fits the radial profile much better. The horizontal, dotted line corresponds to the background field star level, which is $\sim 9 \operatorname{stars~}^{\mathrm{pc}^{-2}}$. Statistical errors are shown.

where $f_{0}$ is the core concentration at radius zero, $r_{\mathrm{c}}$ the core radius, and $a$ a constant for the background offset. As seen in Fig. 5, both models describe the density distribution fairly well. However, the $r^{-1}$ model has a better overall fit (reduced $\chi^{2}=1.2$ ) as compared to the King's model (reduced $\chi^{2}=3.3$ ). Several studies have shown that young embedded clusters can be fitted by $r^{-1}$ profiles (e.g. McCaughrean \& Stauffer 1994; Lada \& Lada 1995) or, by both the inverse radius model and the King's model (e.g. Horner et al. 1997; Teixeira et al. 2004; Baba et al. 2004). As discussed in Baba et al. (2004), the $r^{-1}$ dependence is likely to be reminiscent of the parental cloud core, whereas the King's profile represents systems in dynamical equilibrium. Hence, the better fitting of the $r^{-1}$ model could suggest that the cluster associated with IRAS $06055+2039$ is not yet in complete dynamical equilibrium.

Within errors, the cluster profile merges with the field star level at $\sim 85^{\prime \prime}$, which translates to $\sim 1.1 \mathrm{pc}$ at a distance of $2.6 \mathrm{kpc}$. We take this as the cluster radius. The background level as es-

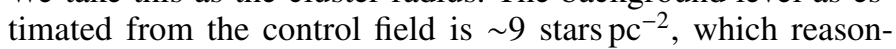
ably agrees with the value of $11.8 \pm 1.6 \mathrm{stars}_{\mathrm{pc}}^{-2}$ yielded by the King's profile fitting. The King's profile fitting also gives a core radius $r_{\mathrm{c}} \sim 0.1 \mathrm{pc}$. The core radius is a scale parameter that depends mainly on cluster parameters like density, luminosity, total mass, etc. Several studies have shown that the core radius of the cluster is also correlated with its age. In their study of young clusters in the LMC, Elson et al. (1989) show that the core radii increase between $\sim 10^{6}$ and $10^{9} \mathrm{yr}$ and then begin to decrease again. Such trends in core radius evolution has also been discussed by Wilkinson et al. (2003) for the LMC clusters and Mackey \& Gilmore (2003) for clusters in the SMC. These authors have shown that apart from the general increasing trend, the spread in the core radii also increases with the age of the cluster. Teixeira et al. (2004) and Baba et al. (2004) derive core radius values of 0.05 and $0.08 \mathrm{pc}$ for the clusters NGC 2316 and RCW 36, respectively. The age estimate for both these clusters is 2-3 Myr. For a comparatively older (5-10 Myr) cluster NGC 2282, Horner et al. (1997) obtain a core radius of $0.19 \mathrm{pc}$. The above values of core radii seem to suggest that the cluster associated with IRAS $06055+2039$ is likely to be the same age ( 2-3 Myr) as NGC 2316 and RCW 36.

The number of stars detected in the $K_{\mathrm{s}}$ band within the $85^{\prime \prime}$ radius of the cluster is 114 . The total background population is 34 [ $=9$ (background) $\times \pi \times(1.1)^{2}$ (area of cluster) $]$. The total

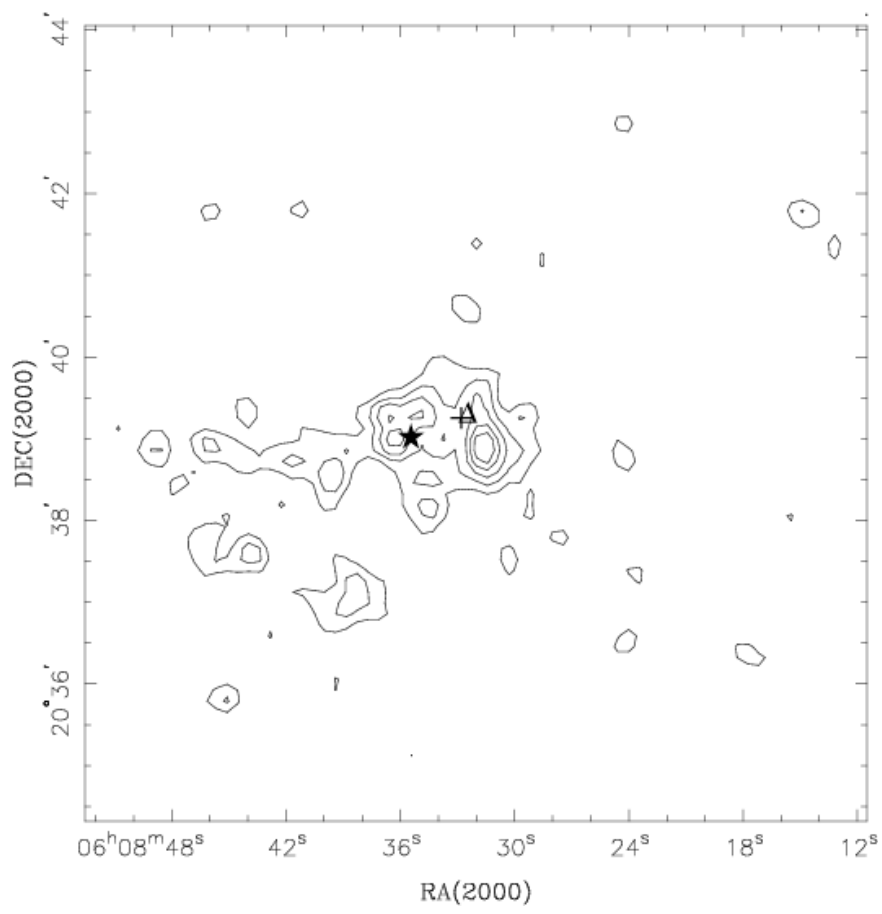

Fig. 6. The contour map of the stellar surface number density obtained by counting stars in a $10^{\prime \prime} \times 10^{\prime \prime}(\sim 0.1 \times 0.1 \mathrm{pc})$ grid for the cluster associated with IRAS $06055+2039$. The contours are from 30 to 140 stars pc $^{-2}$ in steps of $20 \mathrm{stars} \mathrm{pc}^{-2}$. The lowest contour is at the $3 \sigma$ level. The positions of the IRAS point source (plus), the radio peak (open triangle), and the sub-mm peak (star) are shown in the figure.

number of cluster members is thus estimated to be 80 . This

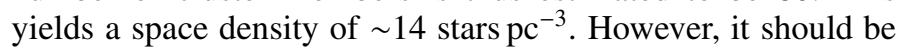
noted here that this density is a lower limit, as we are not completely sampling the stellar population at fainter magnitudes due to the 2MASS sensitivity limits.

Figure 6 shows the surface density map of the region around IRAS 06055+2039. We see the presence of two peaks. The prominent peak that lies to the west coincides spatially with the central, bright source. The secondary peak, which lies $\sim 50^{\prime \prime}$ to the east of the main peak, coincides with the departure seen in the surface density profile from the model profiles around a radial distance of $\sim 0.6 \mathrm{pc}$. This secondary peak is situated close to the edge of the sub-mm peaks presented in Fig. 3. The stellar surface density distribution exhibits a centrally condensed-type structure rather than a hierarchical-type structure (Lada \& Lada 2003). This is consistent with the fact that the derived radial profile fits reasonably well both to the King's model and to the inverse radius model. Hierarchical-type complexes do not follow any well defined profile. Though at a less significant level, centrally condensed clusters have also been seen to show the presence of structures. For example, Lada \& Lada (1995) show the presence of satellite subclusters in the outer regions of IC 348. This is consistent with the double-peaked structure that we see for the cluster associated with IRAS $06055+2039$.

\subsubsection{Colour-colour (CC) and colour-magnitude (CM) diagrams}

The $(H-K)$ versus $(J-H) \mathrm{CC}$ diagram for the cluster associated with IRAS $06055+2039$ is shown in Fig. 7, where we have plotted 53 sources with good quality photometric magnitudes in all three $J H K_{\mathrm{s}}$ bands (2MASS “read-flag" value of 1-3). 


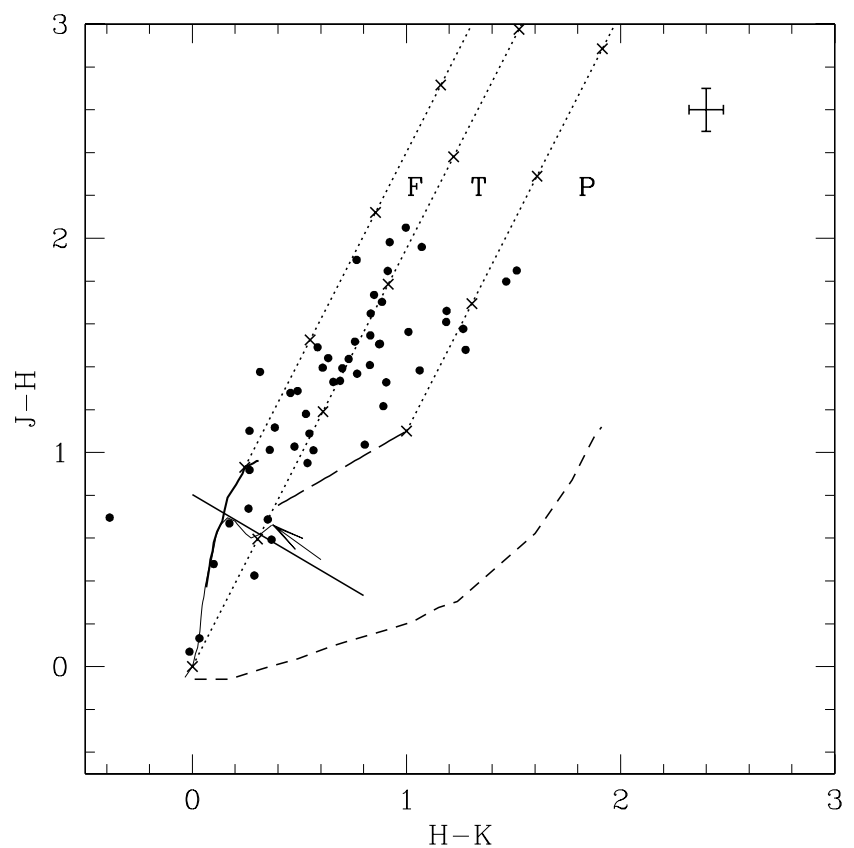

Fig. 7. Colour-colour diagram of the infrared cluster in the IRAS 06055+2039 region. The two solid curves represent the loci of the main sequence (thin line) and the giant stars (thicker line) derived from Bessell \& Brett (1988). The long-dashed line is the classical T Tauri locus from Meyer et al. (1997). The parallel dotted lines are reddening vectors with the crosses placed along these lines at intervals corresponding to five magnitudes of visual extinction. We have assumed the interstellar reddening law of Rieke \& Lebofsky (1985) $\left(A_{J} / A_{\mathrm{V}}=0.282\right.$; $A_{H} / A_{\mathrm{V}}=0.175$ and $\left.A_{K} / A_{\mathrm{V}}=0.112\right)$. The short-dashed line represents the locus of the Herbig AeBe stars (Lada \& Adams 1992). The plot is classified into three regions namely "F", "T", and "P" (see text for details). The colours and the curves shown in the figure are all transformed to the Bessell \& Brett (1988) system. The solid line shown is drawn tangentially to the turn-off point of the main sequence locus. The arrow points to the position corresponding to the central brightest star in the cluster. The mean photometric errors are shown in the upper right corner.

Henceforth, to analysis 2MASS data, we only use good quality photometric data that have the above "read-flag" values. For clarity we have classified the CC diagram into three regions (e.g. Sugitani et al. 2002; Ojha et al. 2004a,b). The "F" sources are located within the reddening bands of the main sequence and the giant stars. These sources are generally considered to be either field stars, Class III objects, or Class II objects with small NIR excess. "T" sources populate the region redward of the "F" region but blueward of the reddening line corresponding to the red end of the T Tauri locus. These sources are classical T Tauri stars (Class II objects) with large NIR excess or Herbig AeBe stars with small NIR excess. Redward of the "T" region is the "P" region, which has mostly protostar-like Class I objects and Herbig AeBe stars. The majority of the sources in our sample are almost equally distributed in the " $F$ " and the "T" regions, whereas only four lie in the "P" region. A total of 18 out of 80 (22\%) sources show infrared excess (i.e. sources populating the "T" and the "P" regions). However, it is important to note here that this NIR excess fraction is just the lower limit as several cluster members detected in the $K_{\mathrm{s}}$ band were not detected in the other two shorter wavelength bands and hence are not part of this sample. The NIR excess in PMS stars is due to the optically-thick circumstellar disks/envelopes. These disks/envelopes become optically thin with age so the fraction of NIR excess stars decreases with

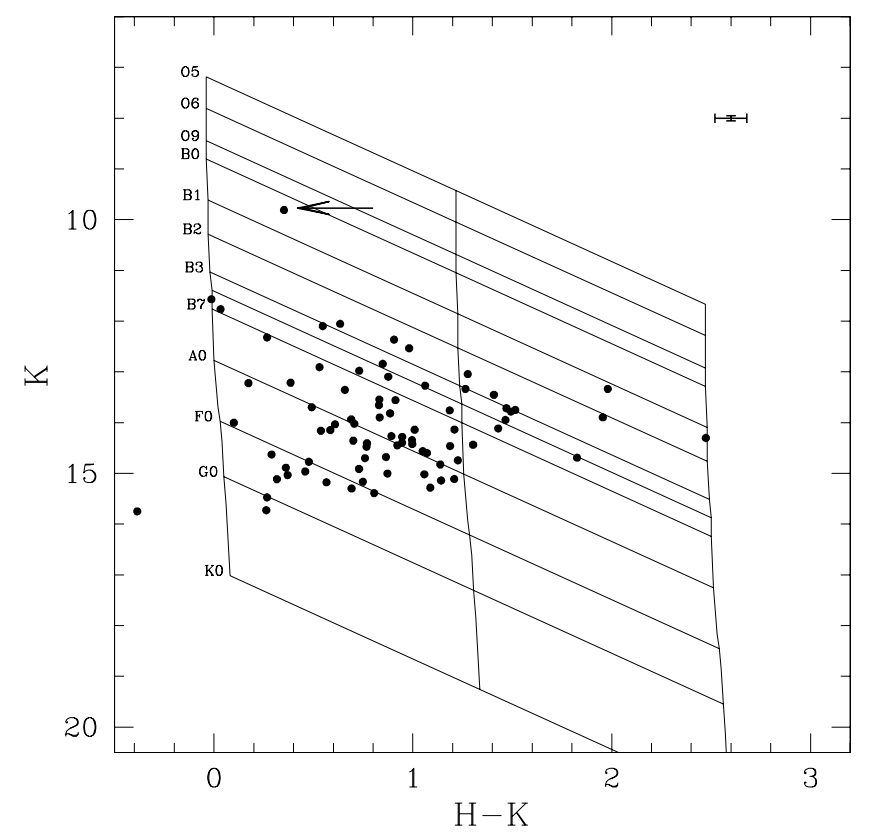

Fig. 8. Colour-magnitude diagram of the infrared cluster in the IRAS 06055+2039 region. The nearly vertical solid lines represent the zero age main sequence (ZAMS) loci with 0, 20, and 40 mag of visual extinction corrected for the distance. The slanting lines show the reddening vectors for each spectral type. The magnitudes and the ZAMS loci are all plotted in the Bessell \& Brett (1988) system. The arrow points to the position corresponding to the central, brightest star in the cluster. The mean photometric errors are shown in the upper right corner.

age. For very young ( $\leq 1 \mathrm{Myr}$ ) embedded clusters the fraction is $\sim 50 \%$ (Lada et al. 2000; Haisch et al. 2000) and it decreases to $\sim 20 \%$ for more evolved (2-3 Myr) clusters (Haisch et al. 2001; Texeira et al. 2004). The fraction of stars with NIR excess seen in this cluster suggests an upper limit 2-3 Myr on the age, which is reasonably consistent with the value suggested from the core radius value. The age estimates for the cluster NGC 2175 associated with the extended HII region Sh 252 are 2 Myr (Grasdalen \& Carrasco 1975) and 1-2 Myr (Kömpe et al. 1989), which agrees rather well with our estimates for the cluster associated with IRAS $06055+2039$, which also belongs to the Sh 252 complex.

We calculated the extinction by de-reddening the stars in the CC diagram. They are shifted to a line drawn tangential to the turn-off point of the main-sequence locus (see Fig. 7). The amount of shift gives an estimate of the extinction of individual stars. The extinction values range from $A_{\mathrm{V}} \sim 0$ to 13 mag with an average foreground extinction of $A_{\mathrm{V}} \sim 7 \mathrm{mag}$. The range of extinction values obtained shows up as the spread of stars along the reddening band in the CC diagram. This indicates that the cluster is partially embedded (Teixeira et al. 2004). Also according to Lada \& Lada (2003), low extinction values ( $\left.A_{\mathrm{V}} \sim 1-5 \mathrm{mag}\right)$ are typical of partially embedded clusters.

Figure 8 shows the $(H-K)$ versus $K$ colour-magnitude (CM) diagram for 79 sources with good quality $H K$ magnitudes. Using the zero-age main-sequence (ZAMS) loci and the reddening vectors, we estimated the spectral type of the brightest star in the cluster to be $\sim \mathrm{B} 0.5$. This is the central IRAS point source and from the NIR estimates seems to be the most massive star in the cluster. A similar estimate for the spectral type is also obtained from analysing the $(J-H)$ versus $J$ CM diagram. 

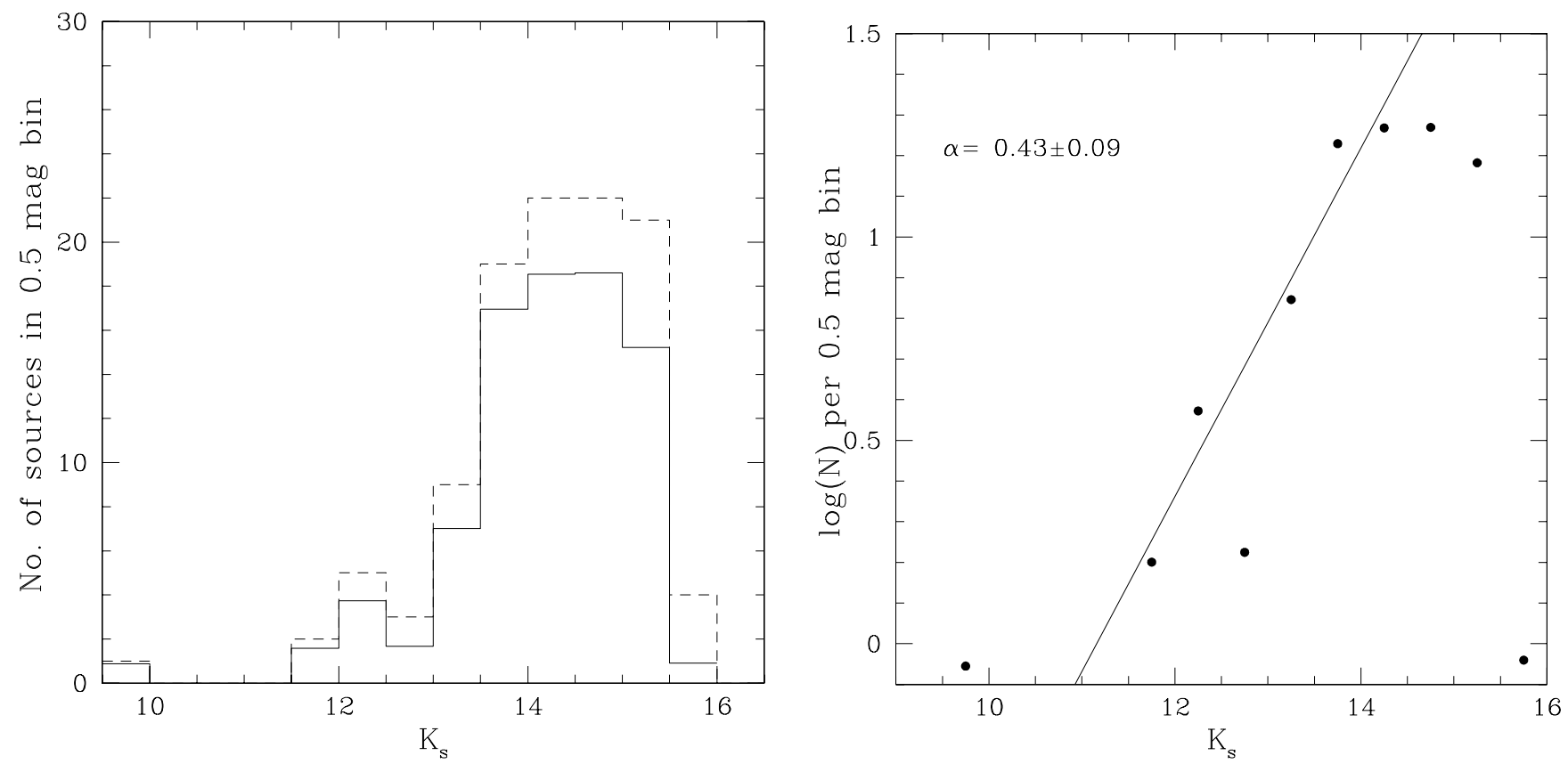

Fig. 9. Left: the corrected $K_{\mathrm{s}}$-band luminosity function (KLF) for the cluster around IRAS $06055+2039$ is shown (solid line). The dotted line is the luminosity function without foreground/background correction. Right: the KLF shown as $\log N$ versus the $K_{\mathrm{s}}$ magnitude. The straight line is the least-square fit to the data points in the magnitude range 11.5-14.5.

\subsection{3. $K_{\mathrm{s}}$-band luminosity function}

We used the 2MASS $K_{\mathrm{s}}$-band star counts to derive the $K_{\mathrm{s}}$-band luminosity function (KLF) for the embedded cluster. In order to obtain the KLF of the cluster, it is essential to account for the background and foreground source contamination. For this purpose we used both the Besançon Galactic model of stellar population synthesis (Robin et al. 2003) and the observed control field star counts. We used the same control field as described in Sect. 4.1.1.

Star counts were predicted using the Besançon model in the direction of the control field. We checked the validity of the simulated model by comparing the model KLF with that of the control field and found that both the KLFs match rather well. As mentioned in the previous section, the average foreground extinction was determined to be $A_{\mathrm{V}} \sim 7 \mathrm{mag}$. Hence, assuming spherically symmetric geometry, the background population is then seen through a cloud with extinction upto $A_{\mathrm{V}} \sim 14 \mathrm{mag}$ $(7 \times 2)$. Model simulations with $A_{\mathrm{V}}=0$ mag and $d<2.6 \mathrm{kpc}$ gives the foreground contamination. The background population was generated with $A_{\mathrm{V}}=14 \mathrm{mag}$ and $d>2.6 \mathrm{kpc}$. We determined the fraction of the contaminating stars (foreground + background) over the total model counts. This fraction was used to scale the observed control field and subsequently the star counts of the modified control field were subtracted from the KLF of the cluster to obtain the final corrected KLF which is shown in the left panel of Fig. 9.

The right panel of Fig. 9 shows the KLF plotted as $\log N$ versus the $K$ magnitude. The KLF can be written as a power-law

$\frac{\mathrm{d} N\left(K_{\mathrm{s}}\right)}{\mathrm{d} K_{\mathrm{s}}} \propto 10^{\alpha K_{\mathrm{s}}}$

where the left side of the equation denotes the number of stars per unit magnitude bin and $\alpha$ is the slope of the powerlaw. A linear least-square fitting algorithm is used to fit the above power law to the KLF in the magnitude range $K_{\mathrm{s}}=$ 11.5 to 14.5 . We obtain a value of $\alpha=0.43 \pm 0.09$ for the cluster.
The least-square fitting is done taking the statistical errors on individual data points.

Within the quoted errors, the estimate of the power-law slope is consistent with the average value of slopes $(\alpha \sim 0.4)$ obtained for other young clusters (Lada et al. 1991; Lada \& Lada 1995; Lada \& Lada 2003). The power-law slope values obtained for other embedded clusters like W3 main and NGC 7538 are significantly lower ( $\alpha \sim 0.17$ to 0.33 - Ojha et al. 2004a,b; Balog et al. 2004). However, it should be noted here that these clusters are much younger $(\lessgtr 1 \mathrm{Myr})$ and the surveys are deeper $\left(K_{\mathrm{s}} \leq 17.5\right)$ that probe the low mass stellar population down to $\sim 0.1 M_{\odot}$.

We estimated the masses of the sources in the cluster by comparing them with the evolutionary models of Palla \& Stahler (1999). Figure 10 shows the $(J-H)$ versus $J C M$ diagram for the cluster field. We used the $J$-band magnitudes rather than $K_{\mathrm{s}}$ because it is less affected by emission from circumstellar material. The solid curve represents the ZAMS isochrone for a 2 Myr cluster from Palla \& Stahler (1999). The Majority of sources have a typical mass of $\sim 2 M_{\odot}$, which we assume to be representative of the stellar population in the cluster. For a log-normal IMF, the power-law slope $(\gamma)$, which is 1.35 for a Salpeter IMF, is variable and is given by $\gamma=0.94+0.94 \log \left(m_{\star}\right)$, where $m_{\star}$ is the stellar mass (Miller \& Scalo 1979; Lada et al. 1993). Using this relation, we estimated $\gamma \sim 1.2$ for this mean mass. The powerlaw slope for the mass to luminosity relation is $\beta \approx 1$ for clusters of age $\sim 10^{6} \mathrm{Myr}$ (Simon et al. 1992; Lada et al. 1993). The corresponding slope of the KLF, $\alpha(=\gamma / 2.5 \beta)$, is 0.48 , which is consistent with the value obtained from the least-square fit to the KLF. As is seen from Fig. 10, the masses of the majority of sources in the cluster are below $2.5 M_{\odot}$ and the lowest mass limit is $\sim 0.4 M_{\odot}$ from our sample.

\subsection{Spatial distribution of UIBs from MSX data}

We used the scheme developed by Ghosh \& Ojha (2002) to extract the contribution of UIBs (due to the PAHs) from the midinfrared images in the four MSX bands. The emission from each 


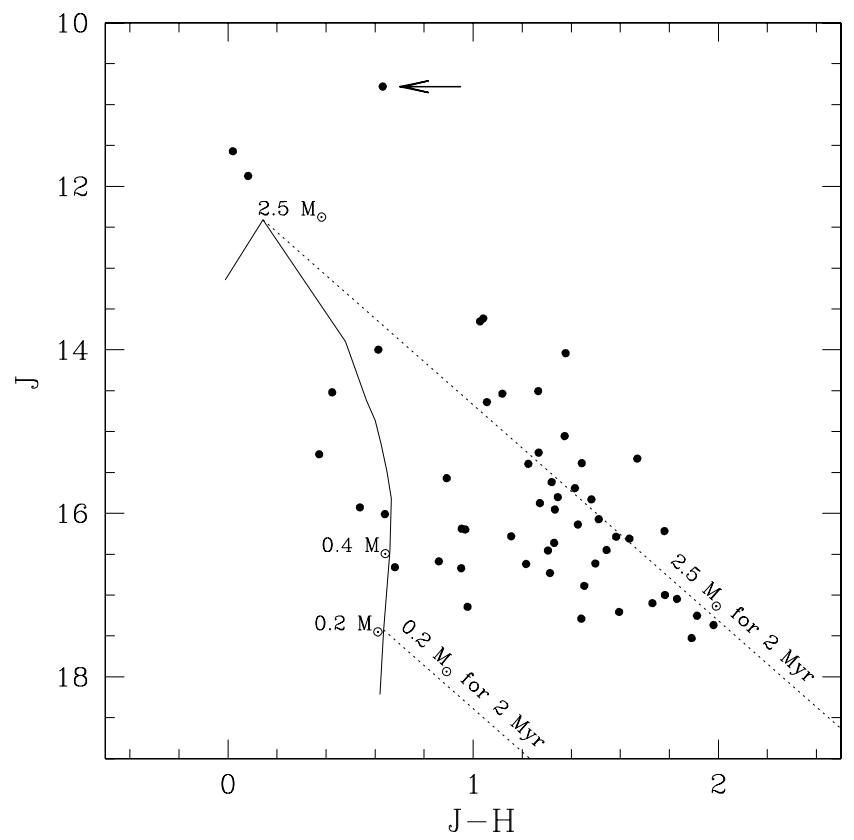

Fig. 10. The mass spectrum for the cluster associated with IRAS 06055+2039. The solid curve represents the model isochrone from Palla \& Stahler (1999) for a 2 Myr PMS stellar population for the mass range $0.1-3 M_{\odot}$. The slanted dotted lines are the reddening vectors for 2.5 and $0.2 M_{\odot}$ PMS stars, respectively. Sources that lie above the reddening vector for $2.5 M_{\odot}$ are luminous massive stars. The arrow points to the position corresponding to the central massive star in the cluster.

pixel is assumed to be a combination of two components. The first is the thermal continuum from dust grains (gray body) and the second is the emission from the UIB features falling within the MSX bands. The scheme assumes that dust emissivity follows the power law of the form $\epsilon_{\lambda} \propto \lambda^{-1}$ and the total radiance due to the UIBs in band $C$ is proportional to that in band $A$. A self consistent non-linear chi-square minimization technique is used to estimate the total emission from the UIBs, the temperature, and the optical depth. The spatial distribution of emission in the UIBs with an angular resolution of $\sim 18^{\prime \prime}$ (for the MSX survey) extracted for the region around IRAS $06055+2039$ is shown in Fig. 11.

Comparing the morphology with the radio continuum maps, we see that the emission from the UIBs is much more extended though the gross morphologies are similar with a relatively steep intensity gradient towards the N-E and a smoothly decreasing intensity distribution to the S-W. The peak position of the UIB distribution matches with the radio peak rather well.

The integrated emission from the region around IRAS $06055+2039$ in the UIB features within the band $A$ of MSX (viz., 6.2, 7.7 and $8.6 \mu \mathrm{m}$ ) is found to be $2.85 \times 10^{-12} \mathrm{Wm}^{-2}$ (see Fig. 11). For comparison, we estimated the emission in individual UIB features from the IRAS LRS spectrum (covering $8-22 \mu \mathrm{m})$, for this source. The total emission in the 7.7 and $8.6 \mu \mathrm{m}$ features is $\sim 7.33 \times 10^{-12} \mathrm{Wm}^{-2}$, in the $11.3 \mu \mathrm{m}$ feature is $\sim 1.32 \times 10^{-12} \mathrm{Wm}^{-2}$, and an upper limit for the $12.7 \mu \mathrm{m}$ feature is $5.9 \times 10^{-13} \mathrm{Wm}^{-2}$. The last value is an upper limit due to possible contamination from the Ne [II] line at $12.8 \mu \mathrm{m}$. Hence, the UIB emission extracted from the MSX band $A$ is $\$ 39 \%$ of the estimate from LRS. This is reasonable considering the larger effective field of view for the latter.

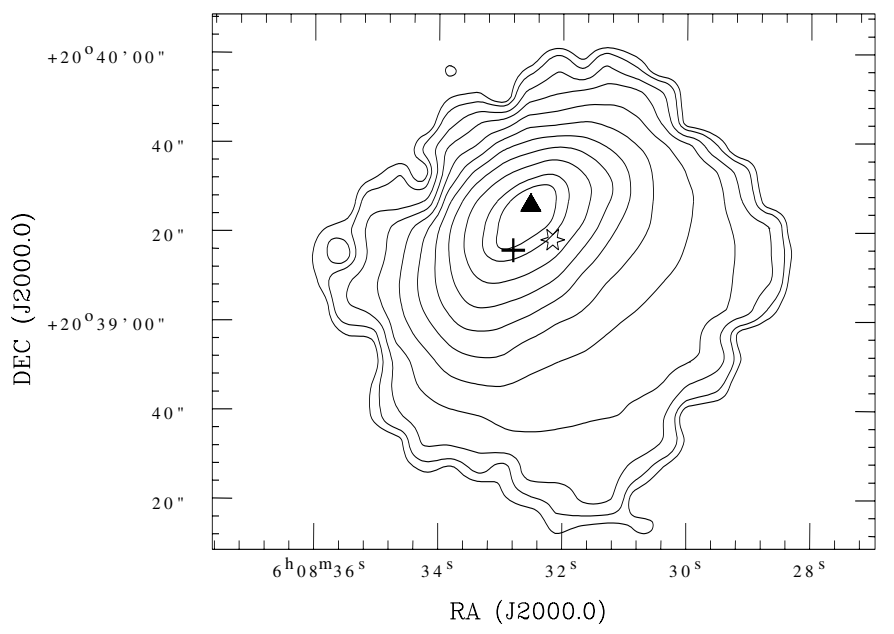

Fig. 11. Spatial distribution of the total radiance in the UIBs for the region around IRAS $06055+2039$ as extracted from the MSX four band images. The contour levels are at 5, 10, 20, 30, 40, 50, 60, 70, 80, 90, and $95 \%$ of the peak emission of $4.1 \times 10^{-5} \mathrm{~W} \mathrm{~m}^{-2} \mathrm{Sr}^{-1}$. The peak position of the UIB distribution (filled triangle), the $1280 \mathrm{MHz}$ radio emission (open star), and the IRAS point source position (plus) are also indicated.

\subsection{Emission from ionized gas}

The radio continuum emission from the ionized gas associated with the region around IRAS $06055+2039$ at 1280 and $610 \mathrm{MHz}$ is shown in Fig. 2. The integrated flux densities from these maps are 183 and $282 \mathrm{mJy}$ at 1280 and $610 \mathrm{MHz}$, respectively. It should be noted here that the flux densities are obtained by integrating up to a $3 \sigma$ level, where $\sigma$ is the rms noise of the maps. The integrated flux densities obtained from the GMRT maps are consistent with the results of Felli et al. (1977) and White \& Gee (1986). Felli et al. (1977) get values of 230 and $205 \mathrm{mJy}$ at 1415 and $4995 \mathrm{MHz}$, respectively. White \& Gee (1986) estimate the total integrated flux density at $5 \mathrm{GHz}$ to be $198 \mathrm{mJy}$.

The contour maps display a cometary morphology with a bright arc-shaped edge on the N-E side and a smoothly decreasing intensity distribution on the opposite side. The cometary morphology is clearly seen in the $610 \mathrm{MHz}$ map. Such a morphology implies that the HII region is ionization bounded towards the N-E and density bounded towards the S-W. The position of the brightest radio peak matches with the central, bright star seen in the infrared images. Similar morphology is seen from the $4995 \mathrm{MHz}$ map of Felli et al. (1977). They report the presence of several dense clumps. According to them the radio continuum emission from this HII region (S252 A) is spatially separate from the larger extended HII region associated with Sh 252. The CO-line observations of Lada \& Wooden (1979) show that this compact HII region (S252 A), the associated $\mathrm{H}_{2} \mathrm{O}$ maser, and $\mathrm{CO}$ bright spot are nearly coincident and located near the interface of the molecular cloud with the extended $\mathrm{S} 252 \mathrm{HII}$ region. The detection of the $\mathrm{H}_{2} \mathrm{O}$ maser also implies the relative youth of the region. Lada \& Wooden (1979) also suggest that S252 A is in the early stages of stellar evolution in which a massive star (or stars) has reached the main sequence and created a compact HII region within its parental molecular cloud. The VLA maps at 15 and $5 \mathrm{GHz}$ of White \& Gee (1986) also trace similar cometary morphologies.

Using the low-frequency flux densities at 1280 and $610 \mathrm{MHz}$ from our GMRT observations and $5 \mathrm{GHz}$ data from White \& Gee (1986), we derived the physical properties of the compact core of the HII region associated with IRAS 06055+2039. 
Mezger \& Henderson (1967) have shown that for a homogeneous and spherically symmetric core, the flux density can be written as

$S=3.07 \times 10^{-2} T_{\mathrm{e}} v^{2} \Omega\left(1-\mathrm{e}^{-\tau(v)}\right)$

where

$\tau(v)=1.643 a \times 10^{5} v^{-2.1}(E M) T_{\mathrm{e}}^{-1.35}$

where $S$ is the integrated flux density in Jy, $T_{\mathrm{e}}$ the electron temperature in $\mathrm{K}, v$ the frequency of observation in $\mathrm{MHz}, \tau$ the optical depth, $\Omega$ the solid angle subtended by the source in steradians, and $E M$ the emission measure in $\mathrm{cm}^{-6} \mathrm{pc}$. Also, $a$ is a correction factor and we used a value of 0.99 (using Table 6 of Mezger \& Henderson 1967) for the frequency range $0.6-5 \mathrm{GHz}$ and $T_{\mathrm{e}}=8000 \mathrm{~K}$. The two GMRT maps were convolved to a common angular resolution of $12^{\prime \prime} \times 12^{\prime \prime}$, which is the resolution of the $5 \mathrm{GHz}$ map of White \& Gee (1986). In our case since, the core is unresolved, $\Omega$ is taken as this synthesized beam size (i.e. $\Omega=1.133 \times \theta_{a} \times \theta_{b}$, where $\theta_{a}$ and $\theta_{b}$ are the half power beam sizes). The peak flux densities of the core in the $0.6-5 \mathrm{GHz}$ frequency range appear to lie in the optically thin region. Using these peak flux densities, we derived the emission measure for an estimated electron temperature.

The electron temperature $\left(T_{\mathrm{e}}\right)$ of HII regions is known to increase linearly with Galacto-centric distance $\left(D_{\mathrm{G}}\right)$ (Deharveng et al. 2000, and references therein), due to the decrease in heavy element abundance with $D_{\mathrm{G}}$, which results in higher a $T_{\mathrm{e}}$. The values of $T_{\mathrm{e}}$, derived by Omar et al. (2002) for a sample of three Galactic HII regions, are also consistent with the relationship given in Deharveng et al. (2000). Assuming $D_{\mathrm{G}}$ as $10 \mathrm{kpc}$ (Shirley et al. 2003) for IRAS 06055+2039 (S252A), we obtained a value of $\sim 8000 \mathrm{~K}$ for the electron temperature. Using this value of the electron temperature, the peak flux densities were used to fit the above equations (Eqs. (3), (4)). The best fit value for the emission measure is $8.8 \pm 0.4 \times 10^{4} \mathrm{~cm}^{-6} \mathrm{pc}$. We obtained an estimate of $1.05 \times 10^{3} \mathrm{~cm}^{-3}$ for the electron density: $n_{\mathrm{e}}=(E M / r)^{0.5}$, with $r$ being the core size which in this case corresponds to the synthesized beam size. These values agree reasonably well with the estimates of Felli et al. (1977) for the brightest peak (A3) of component S252 A (see Fig. 5 of Felli et al. 1977). They derive a value of $9.9 \times 10^{4} \mathrm{~cm}^{-6} \mathrm{pc}$ and $5.95 \times 10^{2} \mathrm{~cm}^{-3}$ for $E M$ and $n_{\mathrm{e}}$, respectively. They assume an electron temperature of $10^{4} \mathrm{~K}$ and a distance of $2 \mathrm{kpc}$. It should also be noted here that the $5 \mathrm{GHz}$ map of Felli et al. (1977) has a larger beam size $\left(\sim 8^{\prime \prime} \times 21^{\prime \prime}\right)$.

Taking the total integrated flux density of $183 \mathrm{mJy}$ at $1280 \mathrm{MHz}$ and using the formulation of Schraml \& Mezger (1969) and the table from Panagia (1973; Table II), we estimated the exciting star of this HII region to be of spectral type B0-B0.5. This is consistent with the spectral class obtained by Felli et al. (1977) and White \& Gee (1986). The FIR flux densities from the IRAS PSC yield a luminosity of $\sim 10^{4} L_{\odot}$, which implies an exciting star of spectral type B0.5, in good agreement with radio measurements.

In addition to the diffuse emission seen in our $1280 \mathrm{MHz}$ map, we also detected a few discrete sources probably representing high-density clumps, which are listed in Table 3 . We designated them as R1, R2,..R7, and their positions are marked in Fig. 2. Three such dense clumps were also detected in the $5 \mathrm{GHz}$ map of Felli et al. (1977). The position of R1 is spatially coincident with the position of the central, bright, and massive $(\sim$ B0.5) star seen in the infrared cluster and is possibly the exciting source of the HII region. The other dense clumps could also
Table 3. Discrete sources extracted from the $1280 \mathrm{MHz}$ map of the region associated with IRAS $06055+2039$.

\begin{tabular}{cccc}
\hline \hline Source & $\begin{array}{c}\text { RA (2000.0) } \\
(\mathrm{h} \mathrm{m} \mathrm{s})\end{array}$ & $\begin{array}{c}\text { Dec (2000.0) } \\
(\mathrm{d} \mathrm{m} \mathrm{s})\end{array}$ & $\begin{array}{c}\text { Peak flux density } \\
(\mathrm{mJy} / \text { beam })\end{array}$ \\
\hline R1 & 060832.16 & +203918.7 & 5.7 \\
R2 & 060831.95 & +203923.1 & 5.4 \\
R3 & 060832.79 & +203916.1 & 4.3 \\
R4 & 060831.04 & +203924.9 & 3.3 \\
R5 & 060830.92 & +203930.3 & 2.8 \\
R6 & 060831.39 & +203902.1 & 3.2 \\
R7 & 060832.20 & +203901.7 & 2.8 \\
\hline
\end{tabular}

be discrete radio sources, but the resolution of our map makes it difficult to comment on their nature. These seven clumps contribute $\sim 5 \%$ of the total integrated emission from the ionized region around IRAS $06055+2039$, the remaining being of a diffuse nature.

\subsection{Emission from shocked neutral gas}

The rotational-vibrational line of molecular hydrogen $\left(\mathrm{H}_{2}\right.$ $(1-0) \mathrm{S} 1,2.12 \mu \mathrm{m})$ traces the shocked neutral gas at the interface between the ionized and the molecular gas. In the photodissociation regions (PDRs), the molecular emission traces the first neutral layer beyond the ionization front. In Fig. 12, we compare the morphologies of the $610 \mathrm{MHz}$ continuum map with the continuum-subtracted narrow-band $\mathrm{H}_{2}$ (left) and $\mathrm{Br} \gamma$ (right) images. It is interesting to note that the shocked molecular hydrogen envelopes the radio emitting region. The $\mathrm{Br} \gamma$ image shows the presence of a faint, diffuse emission that correlates well with the cometary morphology of the radio continuum emission. The $\mathrm{H}_{2}$ arc that traces the ionization front lies beyond the $\mathrm{Br} \gamma$ emission. Comparison with the $1280 \mathrm{MHz}$ continuum map also shows a similar morphology. From the position of the $\mathrm{H}_{2}$ arc, we estimate the radius of the HII region to be $\sim 0.4 \mathrm{pc}$.

\subsection{Emission from dust: temperature, optical depth and dust mass}

As discussed in Sect. 4.2, the MSX images were used to obtain the spatial distribution of the temperature and optical depth $\left(\tau_{10}\right)$ of warm dust with the assumption that the dust is optically thin and the dust emissivity follows a power law of the form $\epsilon_{\lambda} \propto \lambda^{-1}$ (Mathis et al. 1983; Scoville \& Kwan 1976). We obtained peak values of $1.4 \times 10^{-4}$ and $155 \mathrm{~K}$ for $\tau_{10}$ and the dust temperature, respectively. Figure 13 shows the optical depth and the mid-infrared dust temperature maps. Morphologically, the spatial distribution of the UIBs and the optical depth contours are similar with the intensity peaks matching rather well. This indicates the presence of higher dust densities near the embedded cluster. The temperature distribution shows lower values near the centre with a plateau-like feature running diagonally along the $\mathrm{S}-\mathrm{E}$ and N-W direction. Higher temperatures are towards the periphery with the peak seen towards the north. The optical depth and temperature are inherently anti-correlated. Hence, we see that the region has higher optical depth at the centre with the values decreasing outwards. Several peaks seen in the $\tau_{10}$ map could possibly indicate the clumpy nature of the region. As will be evident later, we see similar trends for the $T(12 / 25)$ and $\tau_{25}$ maps derived from the IRAS-HIRES images.

The MSX Point Source Catalog (MSX PSC) lists two mid-infrared sources that fall within the radio nebulosity of IRAS $06055+2039$. We designated them as M1 and M2. The 

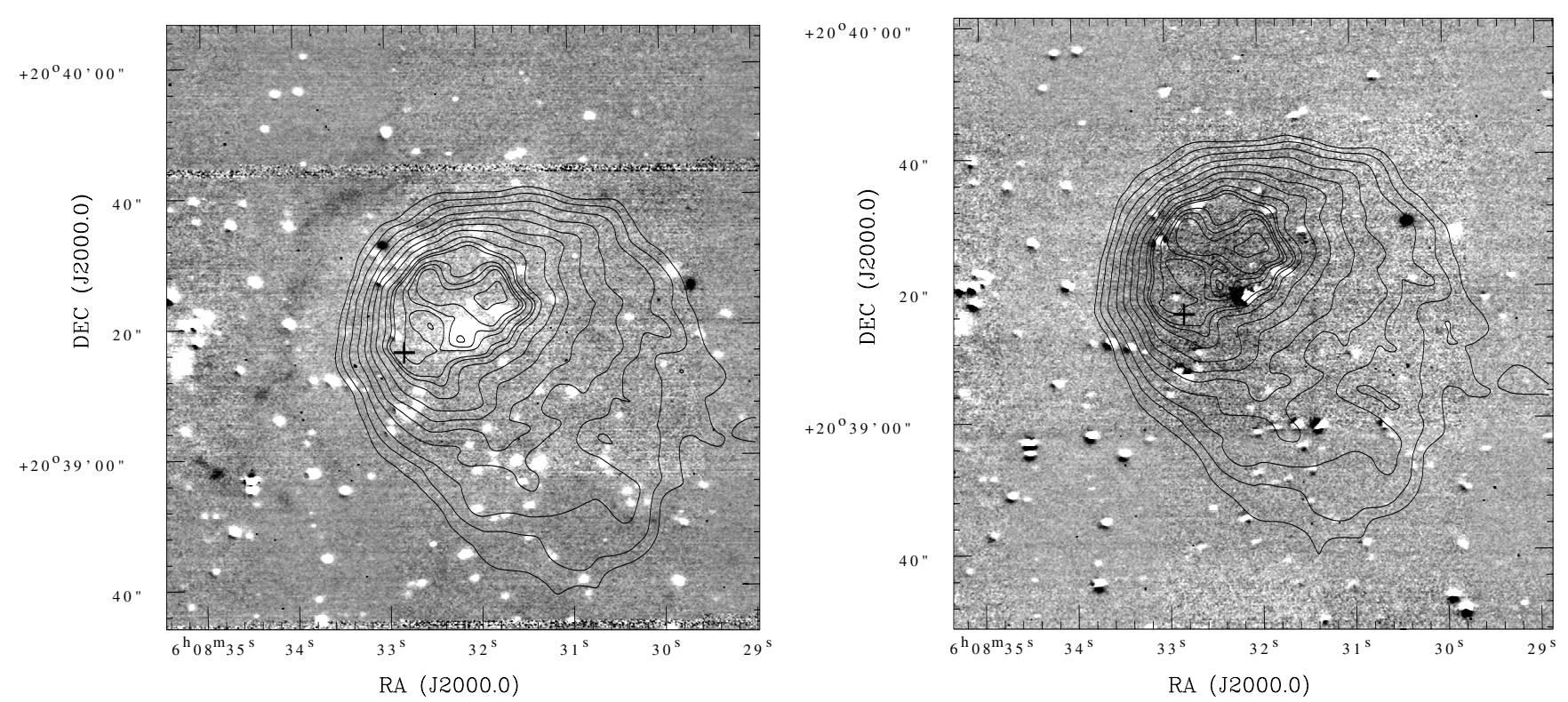

Fig. 12. The $610 \mathrm{MHz}$ radio contours overlaid over the continuum-subtracted $\mathrm{H}_{2}$ (left) and $\mathrm{Br} \gamma$ (right) images for the region associated with IRAS $06055+2039$. The contour levels are the same as in Fig. 2. The plus sign in each image marks the position of the IRAS point source.
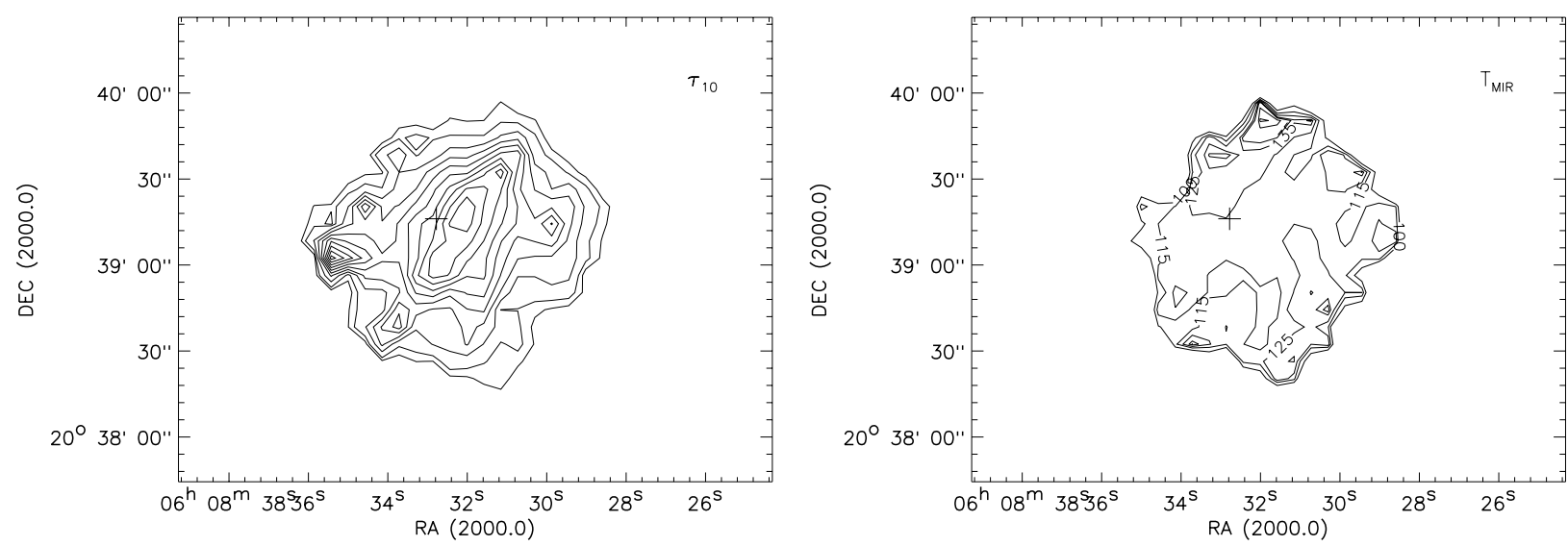

Fig. 13. Left: the spatial distribution of dust optical depth $\tau_{10}$ in the region around IRAS $06055+2039$. The contour levels are at 5, 10, 20, 30, 40, $50,60,70,80$, and $90 \%$ of the peak value of $1.4 \times 10^{-4}$. Right: the spatial distribution of the mid-infrared dust temperature in this region. The contour levels are for the $100,115,125,135,140,150$, and $154 \mathrm{~K}$. The peak temperature is $155 \mathrm{~K}$. The plus sign in each image marks the position of the IRAS point source.

Table 4. Flux densities for the MSX point sources possibly associated with IRAS $06055+2039$.

\begin{tabular}{ccc}
\hline \hline & \multicolumn{2}{l}{ Flux density (Jy) } \\
Wavelength $(\mu \mathrm{m})$ & $\mathrm{M}^{1}$ & $\mathrm{M} 2^{2}$ \\
\hline 8.3 & 3.52 & 0.97 \\
12.1 & 6.24 & 1.40 \\
14.7 & 2.42 & 4.19 \\
21.3 & 6.66 & 13.98 \\
\hline
\end{tabular}

${ }^{1} \mathrm{G} 189.7672+00.3407\left(\alpha_{2000}=06^{\mathrm{h}} 08^{\mathrm{m}} 33.02^{\mathrm{s}} ; \delta_{2000}=20^{\circ} 39^{\prime} 32.04^{\prime \prime}\right)$.

${ }^{2} \mathrm{G} 189.7677+00.3376\left(\alpha_{2000}=06^{\mathrm{h}} 08^{\mathrm{m}} 32.40^{\mathrm{s}} ; \delta_{2000}=20^{\circ} 39^{\prime} 25.20^{\prime \prime}\right)$.

MSX PSC flux densities for these two sources are listed in Table 4. Comparing the MSX mid-infrared colours $F_{21 / 8}, F_{14 / 12}$, $F_{14 / 8}$, and $F_{21 / 14}$ of these two sources with study of the Galactic plane population by Lumsden et al. (2002), we infer that M1 is possibly a Herbig AeBe or a foreground star, whereas M2 falls in the zone occupied mostly by compact HII regions. The NIR counterpart from the $2 \mathrm{MASS}$ catalog for M2 is the central, bright
IRAS point source. The mid- and near-infrared colours $F_{21 / 8}$, $F_{8 / K}, F_{21 / 12}$, and $F_{K / J}$ of this source are also consistent with compact HII regions.

The IRAS-HIRES maps (at 12, 25, 60, and $100 \mu \mathrm{m}$ ) were also used to obtain the spatial distribution of warm and cold dust colour temperatures $(T(12 / 25), T(60 / 100))$ and optical depths $\left(\tau_{25}, \tau_{100}\right)$. We assumed the dust emissivity to follow the power law of the form $\epsilon_{\lambda} \propto \lambda^{-1}$. Figure 14 shows the dust temperature and optical depth maps. The maps for the optical depth $\tau_{25}$ and colour temperature $T(12 / 25)$ represent the warmer dust component. The distribution is centrally dense with the optical depth peak and thus lower derived temperature at the centre. This is similar to the distribution seen in Fig. 13. On the other hand, $\tau_{100}$ and $T(60 / 100)$ distributions are from a relatively colder component that probably forms an envelope around the warmer dust. Unlike the warmer dust temperature distributions (obtained from mid-infrared emission), the $T(60 / 100)$ distribution has its peak at the centre with the temperature decreasing towards the periphery. For $\tau_{25}$ and $\tau_{100}$, we obtained peak values of $2.2 \times 10^{-5}$ and $1.2 \times 10^{-2}$, respectively. The dust temperature distributions 

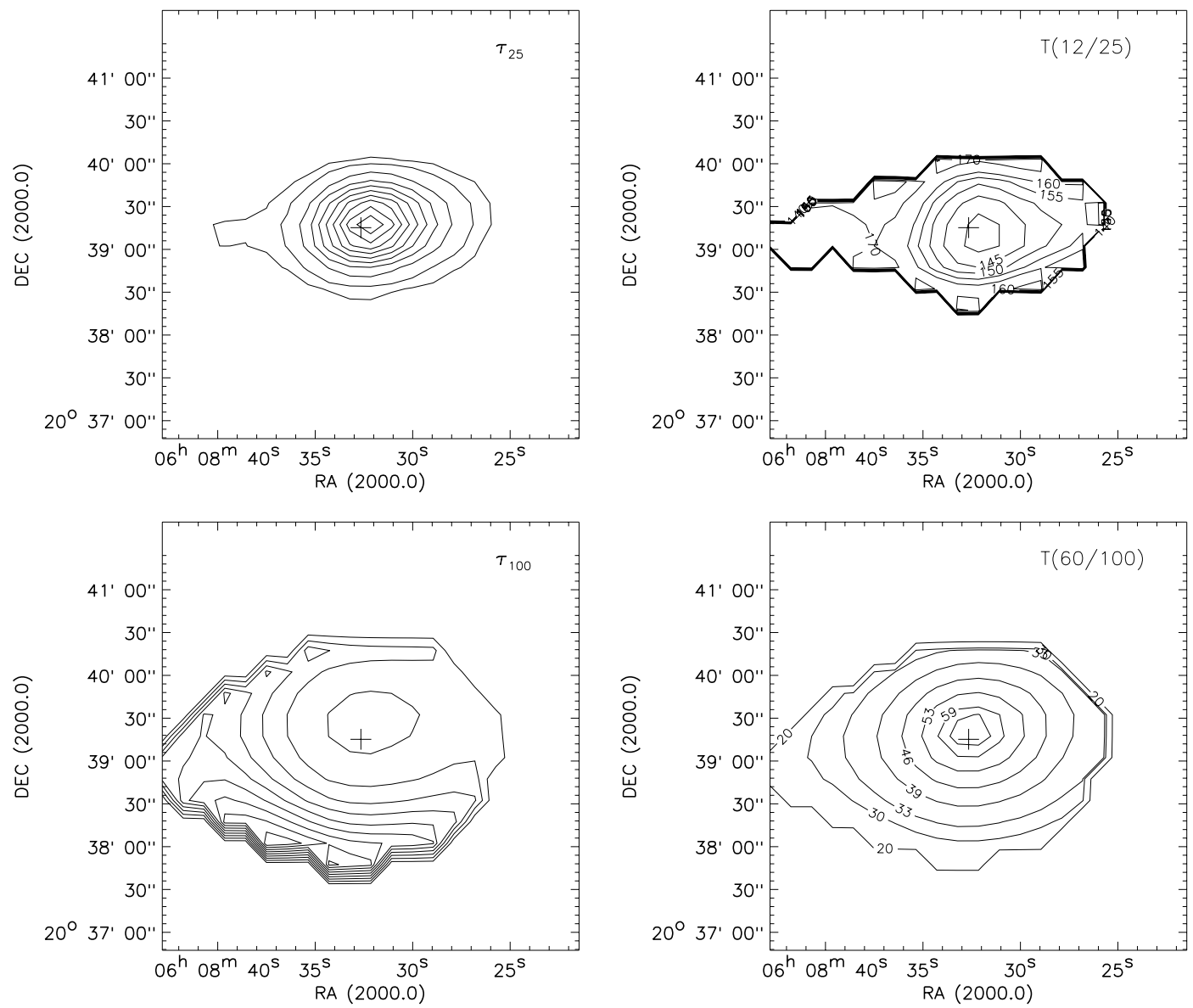

Fig. 14. Upper panel - The dust optical depth $\left(\tau_{25}\right)$ (left) and colour temperature $(T(12 / 25))$ (right) maps of the region around IRAS $06055+2039$. The optical depth contours are $5,10,20,30,40,50,60,70,80,90 \%$ of the peak value of $2.2 \times 10^{-5}$. The dust temperature contour levels are at 140 , $145,150,155,160,170$, and $180 \mathrm{~K}$ from the centre to the periphery. The peak value is $189 \mathrm{~K}$. Lower panel - The dust optical depth $\left(\tau_{100}\right)($ left $)$ and colour temperature $(T(60 / 100)$ ) (right) maps of the region around IRAS $06055+2039$. The optical depth contours are 10, 20, 30, 40, 50, 60, $70,80,90 \%$ of the peak value of $1.2 \times 10^{-2}$ from the centre to the periphery. The dust temperature contour levels are at 20, 30, 33, 39, 46, 53, 59, and $62 \mathrm{~K}$. The peak value is $66 \mathrm{~K}$. The plus sign in each image marks the position of the IRAS point source.

for $T(12 / 25)$ and $T(60 / 100)$ peak at 189 and $66 \mathrm{~K}$, respectively. Schreyer et al. (1996) derive a value of $31.2 \mathrm{~K}$ for $T(60 / 100)$ and $1.21 \times 10^{-3}$ for $\tau_{100}$ from the IRAS PSC flux densities. This difference is due to the different resolution of the raw IRAS and the HIRES-processed maps. Scaling the $\tau_{25}$ peak value, we obtained a value of $\tau_{10} \sim 5.6 \times 10^{-5}$. The difference between this value and the one obtained from the MSX data could be a result of different beam sizes and/or an inhomogeneous medium. Using the peak value of $\tau_{100}$ distribution derived from the IRAS-HIRES maps, we estimated the warm dust mass to be $\sim 6 M_{\odot}$.

We used the emission at submillimetre wavebands to study the cold dust environment in the region around IRAS $06055+2039$. The spatial distribution of the sub-mm emission is shown in Fig. 3. The angular resolutions are 7'. 8 and 15'.2 for the 450 and $850 \mu \mathrm{m}$ wave bands, respectively. The main source (central dense core that covers the region up to $\sim 25 \%$ of the peak intensity) seems elongated in both the maps. Apart from this dense core, several dust clumps are seen that are probably formed due to the fragmentation of the original cloud.

The dust mass can be estimated from the following relation:

$M_{\text {dust }}=1.88 \times 10^{-4}\left(\frac{1200}{v}\right)^{3+\beta} S_{v}\left(\mathrm{e}^{0.048 v / T_{\mathrm{d}}}-1\right) d^{2}$.

This is taken from Sandell (2000) and is a simplified version of Eq. (6) of Hildebrand (1983). The above equation assumes the standard Hildebrand opacities (i.e. $\kappa_{1200 \mathrm{GHz}}=0.1 \mathrm{~cm}^{2} \mathrm{~g}^{-1}$ ). Here, $S_{v}$ is the flux density at frequency $v, T_{\mathrm{d}}$ the dust temperature that we assume to be $20 \mathrm{~K}$ (Klein et al. 2005; Mueller et al. 2002), $\beta$ the dust emissivity index and is taken to be 2 (Hildebrand 1983), and $d$ the distance to the source in kpc. The flux densities are obtained from the JCMT-SCUBA maps shown in Fig. 3. To obtain the flux density of the entire cloud, we integrated up to the last contour (which is at $5 \%$ of the peak value). Using the above relation, we estimate dust masses of $\sim 70$ and $90 M_{\odot}$ from the 450 and $850 \mu \mathrm{m}$ maps, respectively. Assuming a gas-to-dust ratio of 100 , the above values translate to total masses of 7000 and $9000 M_{\odot}$ for the cloud from the 450 and $850 \mu \mathrm{m}$ maps, respectively. We also estimate the total mass of only the central dense core to be $\sim 875$ and $1250 M_{\odot}$ from 450 and $850 \mu \mathrm{m}$ maps, respectively. This source (S252A) has also been studied by Mueller et al. (2002) at $350 \mu \mathrm{m}$ and more recently by Klein et al. (2005) at 850 and $1300 \mu \mathrm{m}$. Scaling to the distance and gas-to-dust ratio assumed by us, the corresponding mass from Mueller et al. (2002) is $\sim 600 M_{\odot}$, and from Klein et al. (2005) is $\sim 100 M_{\odot}$. Comparing the mass derived from the $850 \mu \mathrm{m}$ map, our estimate is very close to the mass obtained by Mueller et al. (2002) considering the fact that they have assumed a higher dust temperature $(29 \mathrm{~K})$. The mass estimate from the $450 \mu \mathrm{m}$ maps is $\sim 35 \%$ lower, which could have been affected by the large atmospheric extinction correction applied to the data. 


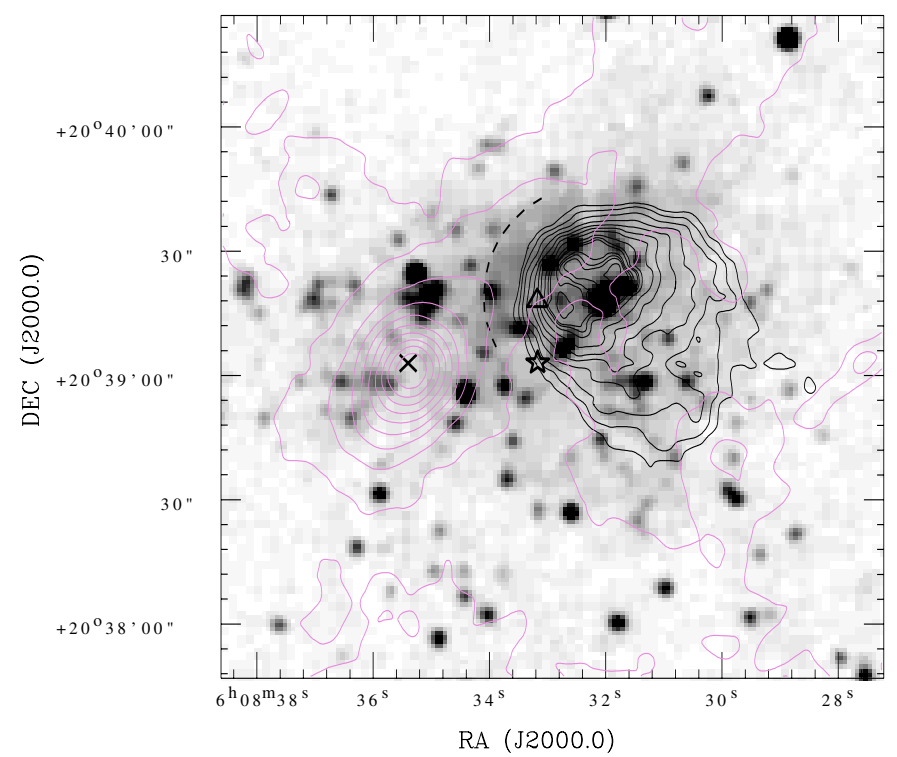

Fig. 15. Contour maps of the emission from the ionized gas at $610 \mathrm{MHz}$ (dark line) and dust emission at $850 \mu \mathrm{m}$ (grey line) are overlaid on the 2MASS $K_{\mathrm{s}}$ band image for the region around IRAS 06055+2039. The dashed arc represents the position of shocked $\mathrm{H}_{2}$. The peak positions of the IRAS-HIRES $12 \mu \mathrm{m}$ (open triangle) and $100 \mu \mathrm{m}$ (open star) maps are indicated. The cross marks the position of the methanol and water masers.

The mass derived by Klein et al. (2005) is relatively lower. This could be due to the fact that the flux density values presented by Klein et al. (2005) are lower compared to our values. From the CS line maps, Zinchenko et al. (1998) derive a cloud mass of $3132 M_{\odot}$. It should be noted here that the CS maps are from a larger region. Furthermore, a point worth discussing here is the effect of varying $T_{\mathrm{d}}$ and $\beta$. Exploring the range of $T_{\mathrm{d}}(20-40 \mathrm{~K})$ and $\beta(1-2)$, we infer that the mass estimates can vary by up to a factor of $\sim 8$.

\subsection{Different components associated with IRAS 06055+2039}

In Fig. 15, we present the various components of the region associated with IRAS $06055+2039$. The plot displays the contour maps of the ionized gas and the emission from dust overlaid on the 2MASS $K_{\mathrm{s}}$-band image. We show the contour plots of $610 \mathrm{MHz}$ radio emission and the $850 \mu \mathrm{m}$ cold dust emission, and mark the peak positions for the 100 and $12 \mu \mathrm{m}$ emission from warm dust. The $850 \mu \mathrm{m}$ emission core lies to the S-E of the ionized region with the warmer dust in between. The 12 and $100 \mu \mathrm{m}$ peaks lie relatively closer to the radio peak. The ionized region is seen to be close to the edge of the molecular cloud. Comparing with Fig. 12, we note that the shocked molecular gas lies between the ionized region and the dense molecular core. The central region of the infrared cluster is located within the HII region that is at the edge of the molecular cloud.

The ionized region and the dense molecular core as seen in the radio and the sub-mm maps, respectively, are possibly at different stages of evolution. The dense molecular core seen in our sub-mm maps does not show any radio emission (down to level of the rms noise which is $0.4 \mathrm{mJy} / \mathrm{beam})$. The sub-mm peak is spatially offset from the FIR peaks and there are no MSX or NIR counterparts seen. This indicates a very early evolutionary stage for this dense and massive molecular core. It is most probably a very early protocluster candidate so we are sampling the initial collapse phase of the star-forming core before the formation of the UCHII region (Williams et al. 2004, and references therein). This fact is further supported by the positions of the water and methanol masers, which are seen to be coincident with the peak of the molecular core. The central peak of the CS map (Zinchenko et al. 1998) and the peaks of the 450 and $850 \mu \mathrm{m}$ JCMT-SCUBA maps match with the position of the masers. More specifically, it is known that methanol maser sites are generally radio quiet (as is the case here) and trace high-mass star-forming protoclusters in very early evolutionary phases (Minier et al. 2005). On the other hand, the ionized region associated with IRAS $06055+2039$, has FIR emission, free-free radio emission, and has NIR and MSX counterparts. This could probably indicate that the source is at a later evolutionary stage between an evolved protocluster and a young cluster. Here, massive stars have started forming and a detectable HII region has been created. The cluster is partially embedded in the parental cloud. The sub-mm emission is weak here. A small subcluster is seen close to the edge of the sub-mm core spatially coincident with the secondary peak mentioned in Sect. 4.1.1. Thus, from this multiwavelength study of the region associated with IRAS $06055+2039$, we see the signatures of different evolutionary stages at different locations.

\section{Summary}

The massive star-forming region associated with IRAS $06055+2039$ has been studied in detail in the infrared, radio, and sub-mm wavelengths, leading to the following conclusions.

1. The morphological details of the environment around IRAS $06055+2039$ show that we have probed the different stages of evolution of star formation present in this cloud complex.

2. High-sensitivity and high-resolution radio continuum maps at 1280 and $610 \mathrm{MHz}$ obtained from our observations using GMRT show interesting cometary morphology. Apart from the diffuse emission, the $1280 \mathrm{MHz}$ continuum map also shows the presence of several discrete sources that probably represent high-density clumps. The total integrated emission implies an exciting star of spectral type B0-B0.5, which is consistent with the estimates derived from NIR colours and IRAS PSC flux densities.

3 The dense molecular cold core was probed with JCMT-SCUBA at 450 and $850 \mu \mathrm{m}$. The sub-mm peak is spatially offset from the peak of radio emission. The sub-mm emission is most probably from a very early protocluster candidate and the radio emission is from a region at a later evolutionary stage where the massive stars have formed an HII region, although the cluster is still partially embedded in the prenatal cloud.

4. In the NIR, the $\mathrm{Br} \gamma$ emission correlates well with the radio continuum emission. The $\mathrm{H}_{2}$ (1-0) S1 line of molecular hydrogen, which traces the first shocked neutral layer beyond the ionization front is seen as an arc towards the N-E of the central IRAS point source and envelopes the ionized emission mapped at radio and NIR wavelengths. The shocked molecular gas lies between the ionized region and the dense cloud core.

5. Using the 2MASS data, we derive a power law slope of $0.43 \pm 0.09$ for the KLF of the NIR cluster associated with this star forming complex. This is consistent with the values obtained for other young embedded clusters. We estimate 
an age of 2-3 Myr for the cluster. The physical structure of the cluster suggests that it is not yet in complete dynamical equilibrium, which is consistent with the scenario of different evolutionary stages seen in the complex.

6. The spatial distribution of the emission from the UIBs as extracted from the MSX images also displays cometary morphology. The total UIB emission from $M S X$ band A is estimated to be $2.85 \times 10^{-12} \mathrm{~W} \mathrm{~m}^{-2}$ and is $\$ 39 \%$ of the emission estimated from the IRAS LRS spectrum. The MSX mid-infrared colours of the central, bright IRAS point source corresponds to a compact HII region.

7. The spatial distribution of temperature and optical depth of interstellar dust has been presented based on the mid- and far-infrared data from the MSX and IRAS (HIRES) surveys. From the derived peak value of the $\tau_{100}$ distribution, we estimate the warm dust mass to be $\sim 6 M_{\odot}$. From the sub-mm emission at 450 and $850 \mu \mathrm{m}$, we estimate the total mass of the cloud to be $\sim 7000-9000 M_{\odot}$.

Acknowledgements. We thank the anonymous referee for the comments and suggestions that helped in improving the paper. We thank the staff at the GMRT who have made the radio observations possible. GMRT is run by the National Centre for Radio Astrophysics of the Tata Institute of Fundamental Research. We thank IPAC, Caltech for providing us the HIRES-processed IRAS products. We thank Annie Robin for letting us use the Besançon Galactic model.

\section{References}

Aumann, H. H., Fowler, J. W., \& Melnyk, M. 1990, AJ, 99, 1674 Baba, D., Nagata, T., Nagayama, T., et al. 2004, ApJ, 614, 818 Balog, Z., Kenyon, S. J., Lada, E. A., Barsony, M., Vinkó, J., \& Gáspár, A. 2004, AJ, 128,

Baudry, A., Desmurs, J. F., Wilson, T. L., \& Cohen, R. J. 1997, A\&A, 325, 255

Bessell, M. S., \& Brett, J. M. 1988, PASP, 100, 1134

Bica, E., Dutra, C. M., \& Barbuy, B. 2003, A\&A, 397, 177

Bronfman, L., Nyman, L.-A., \& May, J. 1996, A\&AS, 115, 81

Carpenter, J. M., Snell, R. L., \& Schloerb, F. P. 1995, ApJ, 450, 201

Chan, S. J., Henning, T., \& Schreyer, K. 1996, A\&AS, 115, 285

Elson, R. A. W., Freeman, K. R., \& Lauer, T. R. 1989, ApJ, 347, L69

Felli, M., Habing, H. J., \& Israël, F. P. 1977, A\&A, 59, 43

Deharveng, L., Pena, M., Caplan, J., \& Costero, R. 2000, MNRAS, 311, 329

Ghosh, S. K., \& Ojha, D. K. 2002, A\&A, 388, 326

Grasdalen, G. L., \& Carrasco, L. 1975, A\&A, 43, 249

Harju, J., Lehtinen, K., Booth, R. S., \& Zinchenko, I. 1998, A\&AS, 132, 211
Haisch, K. E., Lada, E. A., \& Lada, C. J. 2000, AJ, 120, 1396 Haisch, K. E., Lada, E. A., \& Lada, C. J. 2001, AJ, 121, 2065 Hildebrand, R. H. 1983, QJRAS, 24, 267

Horner, D. J., Lada, E. A., \& Lada, C. J. 1997, AJ, 113, 1788

Klein, R., Posselt, B., Schreyer, K., Forbich, J., \& Henning, Th. 2005, ApJS, 161, 361

Kömpe, C., Joncas, G., Baudry, A., \& Wouterloot, G. A. 1989, A\&A, 221,295

Kwok, S., Volk, K., \& Bidelman, W. P. 1997, ApJS, 112, 557

Lada, E. A., Evans, N. J., Depoy, D. L., \& Gatley, I. 1991, ApJ, 371, 171

Lada, C. J., \& Adams, F. C. 1992, ApJ, 393, 278

Lada, C. J., Young, E. T., \& Greene, T. P. 1993, ApJ, 408, 471

Lada, C. J., \& Lada, E. A. 1995, AJ, 109, 1682

Lada, C. J., \& Lada, E. A. 2003, ARA\&A, 41, 57

Lada, C. J., \& Wooden, D. 1979, ApJ, 232, 158

Lada, C. J., Muench, A. A., Haisch, K. E., et al. 2000, AJ, 120, 3162

Lumsden, S. L., Hoare, M. G., Oudmaijer, R. D., \& Richards, D. 2002, MNRAS, 336, 621

Mackey, A. D., \& Gilmore, G. F. 2003, MNRAS, 338, 120

McCaughrean, M. J., \& Stauffer, J. R. 1994, AJ, 108, 1382

Mathis, J. S., Mezger, P. G., \& Panagia, N. 1983, A\&A, 128, 212

Meyer, M. R., Calvet, N., \& Hillenbrand, L. 1997, AJ, 114, 288

Mezger, P. G., \& Henderson, A. P. 1967, ApJ, 147, 471

Miller, G. E., \& Scalo, J. M. 1979, ApJS, 41, 513

Minier, V., Burton, M. G., Hill, T., et al. 2005, A\&A, 429, 945

Mirabel, I. F., Ruiz, A., Rodriguez, L. F., \& Canto, J. 1987, ApJ, 318, 729

Mueller, K. E., Shirley, Y. N., Evans II, N. J., \& Jacobson, H. R. 2002, ApJS, 143, 469

Ojha, D. K., Tamura, M., Nakajima, Y., et al. 2004a, ApJ, 608, 797

Ojha, D. K., Tamura, M., Nakajima, Y., et al. 2004b, ApJ, 616, 1042

Omar, A., Chengalur, J. N., \& Anish Roshi, D. 2002, A\&A, 395, 227

Palla, F., \& Stahler, S. 1999, ApJ, 525, 772

Panagia, N. 1973, AJ, 78, 929

Price, S. D., Egan, M. P., Carey, S. J., Mizuno, D. R., \& Kuchar, T. A. 2001, AJ, 121, 2819

Rieke, G. H., \& Lebofsky, M. J. 1985, ApJ, 288, 618

Robin, A. C., Reylé, C., Derrière, S., \& Picaud, S. 2003, A\&A, 409, 523

Sandell, G. 2000, A\&A, 358, 242

Schraml, J., \& Mezger, P. G. 1969, ApJ, 156, 269

Schreyer, K., Henning, T., Kömpe, C., \& Harjunpää, P. 1996, A\&A, 306, 267

Scoville, N. Z., \& Kwan, J. 1976, ApJ, 206, 718

Shepherd, D. S., \& Churchwell, E. 1996, ApJ, 472, 225

Shirley, Y. L., Evans II, N. J., Young, K. E., Knez, C., \& Jaffe, D. T. 2003, ApJS, 149, 375

Simon, M., Chen, W. P., Howell, R. R., Benson, J. A., \& Slowik, D. 1992, ApJ, 384, 212

Sugitani, K., Tamura, M., Nakajima, Y., et al. 2002, ApJ, 565, L25

Swarup, G., Ananthakrishnan, S., Kapahi, V. K., et al. 1991, Current Sci., 60, 95

Szymczak, M., Hrynek, G., \& Kus, A. J. 2000a, A\&AS, 143, 269

Szymczak, M., Kus, A. J., \& Hrynek, G. 2000b, MNRAS, 312, 211

Teixeira, P. S., Fernandes, S. R., Alves, J. F., et al. 2004, A\&A, 413, L1

White, G. J., \& Gee, G. 1986, A\&A, 156, 301

Wilkinson, M. I., Hurley, J. R., Mackey, A. D., Gilmore, G. F., \& Tout, C. A. 2003, MNRAS, 343,1025

Williams, S. J., Fuller, G. A., \& Sridharan, T. K. 2004, A\&A, 417, 115

Wouterloot, J. G. A., \& Brand, J. 1989, A\&AS, 80, 149

Wu, Y., Wu, J., \& Wang, J. 2001, A\&A, 380, 665

Zinchenko, I., Pirogov, L., \& Toriseva, M. 1998, A\&AS, 133, 337 